\title{
Tanshinone 『A Enhances the Therapeutic Efficacy of Mesenchymal Stem Cells Derived Exosomes in Myocardial Ischemia/reperfusion Injury via Up- regulating miR-223-5p
}

Sheng Li ( $D$ hmh0410@126.com )

Tianjin University of Traditional Chinese Medicine

\section{Lan Li}

Tianjin University of Traditional Chinese Medicine

\section{Rui Guo}

Tianjin University of Traditional Chinese Medicine

\section{Weilong Cao}

Tianjin University of Traditional Chinese Medicine Zhihao Liu

Tianjin University of Traditional Chinese Medicine Jing Zhang

Tianjin University of Traditional Chinese Medicine

Qianqian Liu

Tianjin University of Traditional Chinese Medicine

\section{Yuting Huang}

Tianjin University of Traditional Chinese Medicine

\section{Chuanrui Ma}

First Teaching Hospital of Tianjin University of Traditional Chinese Medicine

\section{Guanwei Fan}

First Teaching Hospital of Tianjin University of Traditional Chinese Medicine

\section{Research Article}

Keywords: exosomes, tanshinone A, mesenchymal stem cells, miR-223-5p, myocardial ischemiareperfusion

Posted Date: November 18th, 2021

DOI: https://doi.org/10.21203/rs.3.rs-1062444/v1 
License: (c) (i) This work is licensed under a Creative Commons Attribution 4.0 International License. Read Full License 


\section{Abstract}

Background: Myocardial ischemia-reperfusion (I/R) injury is a serious obstacle for patients with coronary heart disease to benefit from post-ischemic reflow. After myocardial I/R injury, CCR2 ${ }^{+}$-resident macrophages are rapidly activated and participate in the subsequent inflammatory response, whereas CCR2-resident macrophages play a major role in attenuating cardiac inflammation and promoting tissue repair. Mesenchymal stem cells (MSCs) have gradually become attractive candidates that aid in understanding the pathogenesis and progression of cardiovascular diseases. The low immunogenicity and low carcinogenicity of stem cell-derived exosomes offer advantage in treating myocardial injuries. In this study, we investigated whether MSC-derived exosomes pretreated with tanshinone IIA (TSA) could exhibit stronger cardioprotective function in an I/R rat model and explored its underlying mechanism.

Methods: We investigated the effect of TSA-MSC $\mathrm{exo}_{\mathrm{o}}$ on myocardial I/R injury in vivo. The overexpression of CCR2 in the rat heart was used to determine the regulatory role of CCR2 in I/R injury. High-throughput sequencing of $\mathrm{MSC}_{\text {exo }}$ and TSA-MSC $\mathrm{exo}_{\mathrm{o}}$ to screen differential genes to explore the mechanism of TSA$\mathrm{MSC}_{\text {exo }}$ 's cardioprotective effect.

Results: Compared with $\mathrm{MSC}_{\text {exo }}$, an intramyocardial injection of TSA-MSC $\mathrm{exo}_{\mathrm{o}}$ was found to be more effective in rats in improving cardiac function, limiting the infarct size, inhibiting CCR2 activation, reducing monocyte infiltration and promoting angiogenesis in the heart after myocardial I/R. Moreover, CCR2 had a regulatory effect on monocyte infiltration and angiogenesis after $\mathrm{I} / \mathrm{R}$. Bioinformatics analysis and miRNA sequencing of $\mathrm{MSC}_{\text {exo }}$ and TSA-MSC $\mathrm{Cxo}_{\text {ex }}$ revealed miR-223-5p an effective candidate mediator for TSA-MSC ${ }_{\text {exo }}$ to exert its cardioprotective function and CCR2 as the downstream target.

Conclusion: In summary, our findings indicated that miR-223-5p packaged in TSA-MSC exo $_{\text {inhibited CCR2 }}$ activation to reduce monocyte infiltration and enhanced angiogenesis to alleviate myocardial I/R injury in rats. Thus, the development of an alternative therapy of TSA combined with stem cell-derived exosomes provides an effective strategy for the clinical therapies of ischemic cardiomyopathy.

\section{Background}

Coronary heart disease (CHD) is the main cause of death worldwide and the mortality rate is increasing annually [1]. With the recent popularization of percutaneous coronary intervention (PCl), the affected myocardium of most patients with $\mathrm{CHD}$ can be salvaged through revascularization therapy. However, myocardial damage worsens after reperfusion therapy, resulting in ischemia-reperfusion (I/R) injury [2, 3]. Myocardial I/R injury has become a serious obstacle for patients with $\mathrm{CHD}$ to benefit from reflow after ischemia. During I/R, damage-associated molecular patterns (DAMPs) are released by the injured cardiomyocytes, which induce vascular endothelial cells, immune cells, and fibroblasts to release chemokines, thereby recruiting monocytes in the bone marrow and spleen to infiltrate the ischemic myocardium, hindering myocardial repair, and accelerating deterioration of the heart [4]. Meanwhile, stimulating the growth of small blood vessels and collateral circulation in the ischemic area of the 
myocardium is very important for tissue repair after myocardial injury [5-7]. Hence, studies on inhibiting monocyte infiltration and promotion of angiogenesis after $\mathrm{I} / \mathrm{R}$ have been receiving increased attention.

In mammalian hearts, there are a large number of resident macrophages in the heart tissue that are divided into $\mathrm{CCR}^{-}$and $\mathrm{CCR}{ }^{+}$subsets of embryonic and adult blood lineages [8]. Several studies have confirmed that when myocardial I/R injury occurs, CCR $2^{+}$tissue-resident macrophages are rapidly activated and monocytes are recruited to infiltrate the damaged myocardium to trigger an inflammatory response [9-12], whereas CCR2 ${ }^{-}$tissue-resident macrophages inhibit the recruitment of monocytes $[8,10$, 13-16] and are selectively recruited into the perfused coronary vasculature to stimulate the growth and remodeling of blood vessels to repair the myocardial tissue, thereby improving I/R injury [15]. Therefore, inhibiting CCR2 activation after myocardial damage may effectively reduce the migration and accumulation of inflammatory monocytes to the injured myocardium and may be beneficial in promoting angiogenesis, thus resulting in repair of the myocardial tissue and protecting the injured heart from adverse remodeling. Thus, inhibiting CCR2 activation is of great significance in the treatment of cardiac I/R injury.

In recent years, the development of stem cell regenerative medicine has brought new hope for the treatment of cardiovascular diseases. Several studies have indicated that stem cells can promote the repair of damaged myocardial tissue through paracrine exosomes and cytokines. Exosomes are extracellular vesicles that are 30-150 $\mathrm{nm}$ in diameter. They are responsible for intercellular communication and play a role in the exchange of cellular substances and information. MicroRNA (miRNA) transported by exosomes facilitates cell-to-cell communication through epigenetic regulation of the receiving cell. Additionally, when maternal cells are stimulated by the environment, the secreted exosomal contents (miRNA, mRNA, protein) change and their biological effects are affected due to remodeling of their epigenetic chromatin, thereby enhancing the therapeutic efficacy of exosomes [1719].

Previous studies have shown that TSA can limit infarct size and ameliorate several consequences of myocardial I/R injury, including myocardial enzyme spectrum, oxidation status, inflammation, cardiac dysfunction, and microstructural disorders [20-24]. In this study, we evaluated the cardioprotective effect of TSA-pretreated MSC-derived exosomes (TSA-MSC ${ }_{\text {exo }}$ ) in rats with myocardial I/R injury and explored potential molecular targets. Thus, the development of therapies for exosomes derived from the combination TSA and MSC, and the exploration of therapeutic targets and mechanisms may provide novel strategies for the treatment of patients with heart failure.

\section{Materials And Methods}

\section{Cell culture and TSA pretreatment}

Human umbilical mesenchymal stem cells (HUMSCs) and human umbilical vein endothelial cells (HUVECs) were purchased from Lonza (Walkersville, MD, USA). HUMSCs were cultured in mesenchymal 
stem cell medium (MSCM) (Gibco, MA, USA) supplemented with $10 \%$ fetal bovine serum (FBS) (Gibco) in $5 \% \mathrm{CO}_{2}$ at $37^{\circ} \mathrm{C}$. MSCs from passages 3-5 was pretreated with $10 \mu \mathrm{mol} / \mathrm{L} \mathrm{TSA} \mathrm{(>99 \%} \mathrm{purity)} \mathrm{(Aldrich}$ Chemical Co., USA) in exosome-free MSCM for $24 \mathrm{~h}$ until collection of the medium. HUVECs were cultured in endothelial cell medium (ECM) (Gibco) supplemented with 10\% FBS. Cardiomyocytes (H9C2) was purchased from ATCC (Manassas, VA, USA). H9C2 cells was cultured in Dulbecco's modified Eagle's medium (DMEM) (Gibco) supplemented with 10\% FBS.

\section{Cell transfection}

For cell transfection, MSCs were stably transfected with miR-223-5p inhibitory sequence (i223) containing lentivirus (Genechem, Shanghai, China) or lentivirus containing scrambled control sequences. MSCM was used to prepare a cell suspension with a density of $3-5 \times 10^{4} \mathrm{cells} / \mathrm{mL}$, which was inoculated into a 6-well plate and cultured at $37^{\circ} \mathrm{C}$ for $16-24 \mathrm{~h}$ until cells reached a confluence of $20 \%-30 \%$. The infection reagent and lentivirus were added to MSCs according to the manufacturer's instructions. After $72 \mathrm{~h}$ of infection, the medium was changed to MSCM without exosomes containing $10 \mu \mathrm{mol} / \mathrm{L}$ TSA.

\section{Isolation and characterization of exosomes}

Exosomes were isolated by ultracentrifugation. Briefly, the conditioned supernatants of MSCs pretreated with or without TSA were collected and centrifuged at $300 \times g$ for $10 \mathrm{~min}$. The supernatant was centrifuged at $10000 \times g$ for $30 \mathrm{~min}$ and filtered through a sterile $0.22-\mu \mathrm{m}$ pore filter. The filtered solution was ultracentrifuged at $120,000 \times g$ for $2 \mathrm{~h}$. Lastly, the supernatant was discarded to obtain the exosome precipitate $[25,26]$. The structure of exosomes was observed by transmission electron microscopy (TEM) analysis using a Hitachi HT7700 (Hitachi, Japan) and their size distribution was determined by dynamic light scattering (DLS) using a DynaPro NanoStar (WYATT, USA). The exosomes were identified using western blotting with the marker proteins CD9 (20597-1-AP; Proteintech, Chicago, USA), CD63 (25682-1-AP; Proteintech), and Alix (12422-1-AP; Proteintech). The protein concentration in exosomes was determined using the bicinchoninic acid kit (Thermo Fisher Scientific, Waltham, MA, USA).

\section{Internalization of MSC-derived exosomes in vitro and in vivo}

To evaluate the internalization of MSC-derived exosomes by $\mathrm{H} 9 \mathrm{C} 2$ in vitro, the exosomes were labeled with PKH26 (PKH26PCL; Sigma, USA) according to the manufacturer's protocol. H9C2 $\left(1 \times 10^{5} \mathrm{cell} / \mathrm{sL}\right)$ were cultured in a $5 \% \mathrm{CO}_{2}$ incubator at $37^{\circ} \mathrm{C}$. When the cells attained $80 \%$ confluence, they were supplemented with a medium containing MSC-derived exosomes $(75 \mu \mathrm{g})$ labeled with PKH26. The cells were washed 3 times with phosphate-buffered saline (PBS) after $6 \mathrm{~h}$. The nuclei were stained with DAPI and the cells were observed and photographed using fluorescence microscopy (Leica DM6000M, HE, Germany). After a three-point intramyocardial injection of MSC-derived exosomes (75 $\mu \mathrm{g}$ in $100 \mu \mathrm{L} \mathrm{PBS),}$ the in vivo internalization of PKH26-labeled MSCs-derived exosomes was evaluated. After $0,6,12,24,48$, 
and $72 \mathrm{~h}$, epifluorescence was detected using an IVIS kinetic imaging system (PerkinElmer, Waltham, MA, USA).

\section{In vitro functional assays}

After exosomes were added to HUVECs, a cell scratch test was performed to evaluate the proliferation and migration of endothelial cells in vitro. Next, CD31 (11265-1-AP; roteintech) and vascular endothelial growth factor (VEGF) (19003-1-AP; Proteintech) immunofluorescence were used to evaluate the angiogenesis of HUVECs. ECM was used to prepare a cell suspension with a density of $1 \times 10^{5} \mathrm{cells} / \mathrm{mL}$, which was inoculated into a 6-well plate and cultured in $5 \% \mathrm{CO}_{2}$ at $37^{\circ} \mathrm{C}$ until $90 \%$ confluence was attained. The cells were scratched using a pipette tip and a serum-free medium was added. Then, the cells were taken out at 0 and $24 \mathrm{~h}$ of incubation and photographed. The supernatant was discarded and the cells were fixed with $4 \%$ paraformaldehyde. Goat serum was used to block the activity for 30 min at $25^{\circ} \mathrm{C}$. Next, the cells were incubated overnight with CD31 and VEGF at $4^{\circ} \mathrm{C}$ and subsequently incubated with anti-rabbit horseradish peroxidase (HRP) conjugated to Alexa Fluor 647 (ab190565; Abcam, UK) dye and anti-mouse HRP conjugated to Alexa Fluor 488 (ab171449; Abcam) at $37^{\circ} \mathrm{C}$ in the dark for $1 \mathrm{~h}$. DAPI was used to stain the nucleus and the cells were observed and photographed using fluorescence microscopy.

\section{Establishment of the I/R model and exosome delivery}

All animal procedures were approved by the Tianjin University of Traditional Chinese Medicine Animal Research Committee (TCM-LAEC2014005) and performed in accordance with Directive 2010/63/EU of the European Parliament. Male Sprague-Dawley (SD) rats $(200 \pm 20 \mathrm{~g})$ were purchased from Beijing Weitonglihua Laboratory Animal Technology (Beijing, China). Animals were housed in a specificpathogen-free (SPF) facility based at Tianjin University of Traditional Chinese Medicine at a controlled temperature of $20-25^{\circ} \mathrm{C}$ and humidity of $65-70 \%$ and were subjected to a $12 \mathrm{~h}$ light/12 $\mathrm{h}$ dark cycle. All rats received a standard pellet diet and were provided access to water ad libitum. The rats were randomized into the following treatment groups: (1) sham operation ( $n=15,100 \mu \mathrm{L} P B S),(2) \mathrm{l} / \mathrm{R}$ model

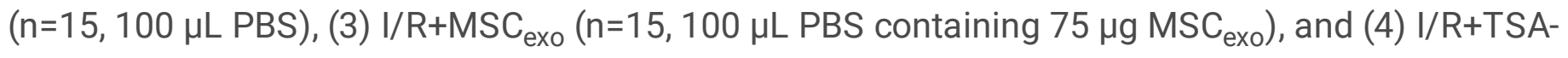
MSC $_{\text {exo }}\left(n=15,100 \mu \mathrm{L}\right.$ PBS containing $75 \mu \mathrm{g}$ TSA-MSC $\left._{\text {exo }}\right)$. For the establishment of the I/R model, rats were anesthetized using an intraperitoneal injection of $5 \%$ tribromoethanol $(30 \mathrm{mg} / \mathrm{kg})$. Thoracotomy was performed in the space between the third and fourth ribs to expose the heart. A 6-0 suture was used to ligate the left anterior descending coronary artery. One hour after ischemia, the suture was removed for reperfusion [27]. Exosomes (75 $\mu \mathrm{g}$ in $100 \mu \mathrm{L}$ PBS) or PBS were injected into the boundary region of the infarcted heart at three different locations. The rats in the sham operation group were not ligated. Specific CCR2 overexpression was achieved in the left ventricles of rats by using adeno-associated virus (AAV)9based delivery vectors (Genechem, Shanghai, China). 
Following tracheal intubation and thoracotomy, AAV9 expressing the CCR2 open reading frame (titer: $1.32 \times 10^{12}$ ) or a scrambled control sequence bearing no homology to known gene transcripts (titer: $1.14 \times 10^{12}$ ) were injected into the left ventricle at multiple sites. The myocardial I/R model was established successfully after 8 weeks of transfection.

\section{Echocardiography to assess left ventricular (LV) function}

Two-dimensional M-mode echocardiography was performed on day 3 post-surgery using Vevo 2100 (VisualSonic, Canada) ultra-high-resolution animal ultrasound imaging system to evaluate LV function after rats were anesthetized by isoflurane. Three representative cycles were captured for each animal and the LV ejection fraction (LVEF) and LV fraction shortening (LVFS) were calculated as described previously [28]. A Millar pressure-volume catheter (SPE-869, \#840-813G) connected to an MPVS Ultra PV circuit system (Millar, Houston, TX, USA) was used to measure the pressure volume of the left ventricle of rats. The catheter entered the left ventricle through the right common carotid artery after calibration and a data acquisition system (LabChart Pro, ADInstruments, Colorado Springs, CO, USA) was used to calculate the measured values of $\mathrm{dP} / \mathrm{dt}$ max and $\mathrm{dP} / \mathrm{dt}$ min.

\section{2,3,5-Triphenyltetrazolium chloride (TTC) staining}

Rats were euthanized by cervical dislocation after 3 days of reperfusion. The heart was isolated and 5 horizontal slices were obtained on average. The tissue slices were incubated in TTC (G3005; Solarbio, Beijing, China) staining solution at $37^{\circ} \mathrm{C}$ in the dark for $30 \mathrm{~min}$, fixed with $4 \%$ paraformaldehyde, and then photographed. As reported previously, the necrotic myocardium was stained white, whereas the viable myocardium was stained red. The images were processed and analyzed using ImageJ ( $\mathrm{NIH}$, Bethesda, MD, USA) [27]. The percentage of infarct area was calculated using the following equation:

\section{Infarct area $(\%)=$ Infarct area $/($ viable area + Infarct area $) \times 100 \%$}

\section{Histological analysis}

Heart tissues were fixed with $4 \%$ paraformaldehyde, embedded in paraffin, cut into $4-\mu m$-thick sections, and stained with hematoxylin/eosin (HE) and Sirius Red and terminal deoxynucleotidyl transferase dUTP nick end labeling (TUNEL) (T2190; Solarbio) according to the manufacturer's instructions. For immunofluorescence co-staining, the heart tissue sections were incubated with CCR2 (ab273050; Abcam), CD68 (66231-2-Ig; Proteintech), a-SMA (67735-1-Ig; Proteintech), and VEGF (66828-2Ig; Proteintech) overnight at $4^{\circ} \mathrm{C}$ and then incubated with anti-rabbit HRP and anti-mouse HRP for $1 \mathrm{~h}$ at 
$37^{\circ} \mathrm{C}$ in the dark. The nuclei were finally stained with DAPI. Images were observed and acquired using fluorescence microscopy (Leica DM6000M, HE, Germany).

\section{Flow cytometry}

According to the manufacturer's recommendations, the enzyme mixture in the Multi-Tissue Dissociation kit 2 (130-110-203; Miltenyi, Germany) was used to digest the cells in the myocardial tissue. Briefly, the heart tissue of the infarct marginal zone was cut into small pieces $\left(1-2 \mathrm{~mm}^{3}\right)$ and suspended in $2.5 \mathrm{~mL}$ of the enzyme mix in gentleMACS C Tubes (130-093-237; Miltenyi). After incubation at $37^{\circ} \mathrm{C}$ for $15 \mathrm{~min}$, the $C$ Tubes were attached onto the sleeve of a gentleMACS Dissociator (Miltenyi), and the program "37C_Multi_G" was run until the myocardial tissue was in a single-cell suspension state. The digestion was stopped with DMEM containing $10 \%$ FBS and the single-cell suspension was centrifuged at $600 \times g$ at $4^{\circ} \mathrm{C}$ for $5 \mathrm{~min}$. After washing with PBS, the cells were incubated with CCR2 (PA5-23040; Thermo Fisher Scientific), major histocompatibility complex class II (MHCII) (17-0920-82; Thermo Fisher Scientific) antibodies at $37^{\circ} \mathrm{C}$ for $1 \mathrm{~h}$ in the dark. Flow cytometry was carried out using a BD LSRFortessa X-20 Cell Analyzer (BD Biosciences, NJ, USA), and FlowJo software (Treestar, OR, USA) was used for data analysis.

\section{Western blotting}

Protein lysate (PMSF: RIPA=1:100) (Solarbio) was used to lyse the heart tissue from the infarct marginal zone. The extracted proteins were subjected to polyacrylamide gel electrophoresis (GenScript, Nanjing, China) and transferred onto polyvinylidene difluoride membranes (Thermo Fisher Scientific). The membranes were blocked with QuickBlock Western reagent (Beyotime, Beijing, China) and incubated sequentially with CCR2 overnight at $4^{\circ} \mathrm{C}$. Subsequently, the washed membranes were incubated with the corresponding HRP-conjugated secondary antibodies (SA00001-2; Proteintech) for $2 \mathrm{~h}$ at $25^{\circ} \mathrm{C}$. Lastly, a gel imaging system (GE Healthcare, $\mathrm{CHI}$, USA) was used to quantify the protein bands. The western blots were quantified using ImageJ software (NIH, MD, USA).

\section{Quantitative polymerase chain reaction (qPCR)}

mRNA and miRNA reverse-transcription kits were used to obtain cDNA following the manufacturer's instructions (Roche, Basel, Switzerland) and SYBR Green PCR master mix (Roche) was used for qPCR. Analysis of gene expression was performed on a CFX96TM PCR detection system (BioRad, Redmond, WA, USA). GAPDH and U6 were used as housekeeping genes to normalize the expression of mRNA and miRNA. The mRNA and miRNA primer sequences were as follows: CCR2 Forward:

CACACTGCTGCGTTAATCCTATC; CCR2 Reverse: TCACTCGGTCTGCTGTCTCC; CCL2 Forward: TCTCTTCCTCCACCACTATGC; CCL2 Reverse: GTTCTCCAGCC 
GACTCATTG; CCL3 Forward: AGAGGCAGCGAGTACCAGTC; CCL3 Reverse:

TAGGAGAAGCAGCAGGCAGTC; CXCL2 Forward: AAAGATACTGAACAAAG

GCAAGG; CXCL2 Reverse: CAGACAGCGAGGCACATC; CXCL3 Forward: TCTGCTGCTTCTGCTGATG; CXCL3 Reverse: TTGAGAGTGGCTATGACTTCT

G; miR-223-5p: GCGCGCGTGTATTTGACAAGCTGAGTT; U6: CCGAGAGAAGATTAGCATGGCCCCTG.

\section{Next-generation sequencing (NGS) of exosomal microRNA}

Total RNA was extracted from $\mathrm{MSC}_{\text {exo }}$ and TSA-MSC $\mathrm{exo}_{\text {of }}$ for reverse transcription and polymerase chain reaction. NGS was outsourced and performed at Ouyi Biomedical Technology (Shanghai, China).

\section{Target gene prediction}

Bioinformatics was used to predict the target genes of miR-223-5p and to explore the relationship between miR-223-5p and CCR2. The principle is that the smaller the free energy of miRNA sequence and mRNA sequence binding, the easier is the binding, thereby playing a regulatory role on target genes. The gene sequences of miR-223-5p and CCR2 mRNA were searched in the NCBI database. Rlsearch 2.0 software was used for target gene prediction to explore the binding between miR-223-5p and CCR2.

\section{Statistical analyses}

Experimental data were statistically processed using SPSS 22.0 software and GraphPad Prism 6 was used for graphical constructions. Data are expressed as mean \pm standard deviation (SD). Multiple groups of samples were compared using one-way ANOVA, if they obeyed normal distribution and homogeneity of variance. A t-test was used to compare differences between groups. $P<0.05$ was considered statistically significant.

\section{Results}

\section{Characterization and internalization of exosomes}

Exosomes were obtained based on the above methods of exosome extraction. TEM findings revealed the TSA-MSC $_{\text {exo }}$ to be round vesicles with a lipid bilayer (Fig. 1A). DLS analysis revealed that particle

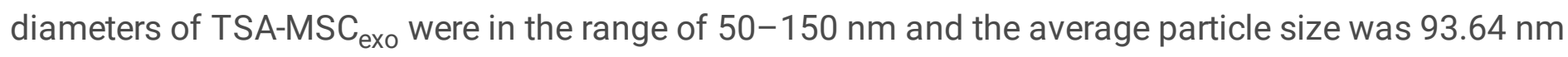
(Fig. 1B). To evaluate the internalization of exosomes by cells, the PKH26-labeled TSA-MSC exo $_{\text {were co- }}$ cultured with $\mathrm{H} 9 \mathrm{C} 2$ cardiomyocytes for $6 \mathrm{~h}$ and observed using fluorescence microscopy (Fig. 1C).

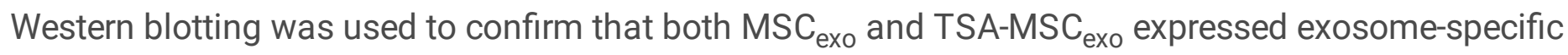


marker proteins CD9, CD63, and Alix (Fig. 1D). PKH26-labeled TSA-MSC $\mathrm{exo}_{\text {ex }}$ was injected into rats through the myocardium in situ to evaluate its residence time in the heart. We found that TSA-MSC exo $_{\text {could be }}$ detected even after $72 \mathrm{~h}$ of injection. The radiation efficiency of TSA-MSC $\mathrm{exo}_{\mathrm{o}}$ was found to decrease gradually within $72 \mathrm{~h}$ (Fig. 1E). These results indicated that the vesicles extracted by ultracentrifugation were indeed exosomes, and whether in vivo or in vitro, exosomes could be internalized by cardiomyocytes, thereby laying the foundation for their therapeutic effects.

\section{Intramyocardial delivery of TSA-MSC exo improved cardiac function and reduced infarct size after myocardial I/R}

A rat model of I/R was used to evaluate the cardioprotective effects of exosomes in vivo. After 3 days of reperfusion, M-mode echocardiographic analysis showed that $\mathrm{MSC}_{\text {exo }}$ and TSA-MSC $\mathrm{exo}_{\text {ex }}$ treatment improved the wall motion amplitude of the LV (Fig. 2A). The LV contractility and function of rats with I/R treated with $\mathrm{MSC}_{\text {exo }}$ and TSA-MSC $\mathrm{exo}_{\mathrm{o}}$ were found to improve based on the significant increase in LVEF and LVFS 3 days after injection. The maximal and minimal left ventricular pressure derivative $(\mathrm{dP} / \mathrm{dt})$ was assessed using invasive hemodynamic measurement of pressure-volume (PV) loops. $\mathrm{MSC}_{\text {exo }}$ and TSA-MSC $_{\text {exo }}$ treatment in rats markedly increased $\mathrm{dP} / \mathrm{dt}$ max and decreased $\mathrm{dP} / \mathrm{dt}$ min compared with

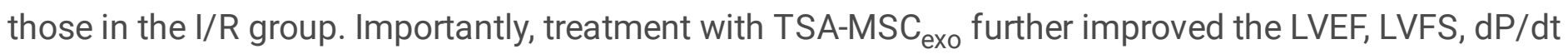
max, and $\mathrm{dP} / \mathrm{dt}$ min after I/R compared with treatment using $\mathrm{MSC}_{\text {exo }}$ (Fig, 2B-E). TTC staining was performed to evaluate the outcome of myocardial infarction after 3 days of injection and to quantify the area of the myocardial infarct. The results showed that the infarct size in rats with I/R treated with

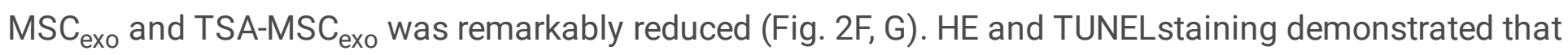
inflammatory cell infiltration and apoptosis in the myocardial tissue of rats with $\mathrm{I} / \mathrm{R}$ were markedly worsened, respectively, and treatment with $\mathrm{MSC}_{\text {exo }}$ and $\mathrm{TSA}-\mathrm{MSC}_{\mathrm{exo}}$ significantly alleviated the occurrence of these conditions. Consistently, treatment with TSA-MSC $\mathrm{exo}_{\text {ox }}$ was found to have significant efficacy (Fig. 2H-K). Taken together, the above findings suggested that TSA-MSC $\mathrm{Cxo}_{\text {ox }}$ exerted a higher cardioprotective effect in rats after I/R than $\mathrm{MSC}_{\text {exo. }}$.

\section{Intramyocardial delivery of TSA-MSC exo $_{\text {inhibited CCR2 }}$ activation, reduced monocyte infiltration, and promoted angiogenesis after I/R}

Studies have confirmed that interrupting the recruitment of CCR2 signals plays a cardioprotective effect in animal models of myocardial injury [29]. Therefore, we investigated the effects of $\mathrm{MSC}_{\text {exo }}$ and TSA$\mathrm{MSC}_{\text {exo }}$ on CCR2 after I/R. The results indicated that the number of $\mathrm{CCR}^{+}$cardiac-resident macrophages and the expression of CCR2 mRNA in rat hearts were significantly increased after I/R injury. Treatment

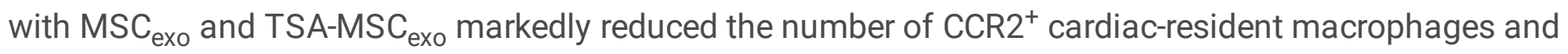

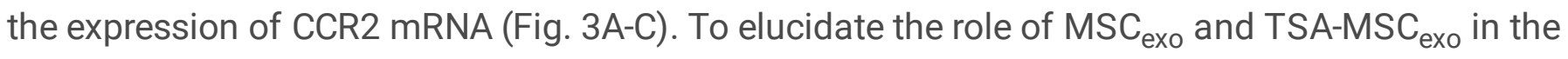


infiltration of monocytes in the heart of rats with I/R injury, we determined changes in the monocyte chemokines in the left ventricular tissue of rats after 3 days of reperfusion. The results suggested that the mRNA expression of CCL2, CCL3, CXCL2, and CXCL3 in rats in the I/R group was significantly increased compared with that in the sham group, and the mRNA expression of CCL2, CCL3, CXCL2, and CXCL3 in rats treated with $\mathrm{MSC}_{\text {exo }}$ and TSA-MSC $\mathrm{exo}_{\mathrm{o}}$ declined markedly (Fig. 3D-G). Monocytes, which are the circulating precursors of macrophages [30]. On entry into tissues, monocytes give rise to macrophages [31]. Therefore, CD68 was used as a marker of macrophages to assess the infiltration of monocytes. Immunofluorescence studies were conducted using tissue sections of rat hearts 3 days after reperfusion. The expression of CCR2 and CD68 in the I/R group increased significantly compared with that in the

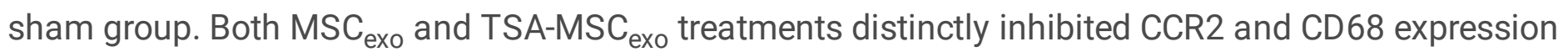
(Fig. $3 \mathrm{H}-\mathrm{J}$ ). After myocardial I/R occurs, the circulating monocytes are recruited to the myocardium to induce inflammation, thereby enhancing protease activity and triggering angiogenesis. The continuous development of inflammation hinders myocardial tissue repair. However, angiogenesis is a phenotype of tissue repair [31]. To determine the efficacy of $\mathrm{MSC}_{\text {exo }}$ and TSA-MSC $\mathrm{exo}_{\mathrm{O}}$ on angiogenesis in rats with I/R injury, immunofluorescence studies were performed using the tissue sections of rat hearts 3 days after reperfusion. The results showed that $\mathrm{MSC}_{\text {exo }}$ and $\mathrm{TSA}^{-\mathrm{MSC}_{\text {exo }}}$ treatment downregulated the expression of CCR2 and upregulated the expression of vascular smooth muscle cell marker, a-SMA as well as VEGF, compared with that in the I/R group (Fig. 3K-0). Sirius Red staining analysis demonstrated that the treatment of $\mathrm{MSC}_{\text {exo }}$ and TSA-MSC $\mathrm{Mxo}_{\text {ex }}$ markedly reduced the collagen deposition in the myocardial tissue of I/R rats to alleviated adverse ventricular remodeling (Fig. 3P, Q). In addition, results from the endothelial cell scratch experiments showed that the number of newborn endothelial cells increased and the scratch distance between endothelial cells decreased significantly after treatment with $\mathrm{MSC}_{\text {exo }}$ and TSA-MSC $_{\text {exo }}$ when scratches at 0 and $24 \mathrm{~h}$ were compared (Fig. 3R, S). This finding indicated that $\mathrm{MSC}_{\text {exo }}$

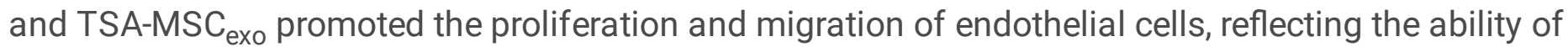

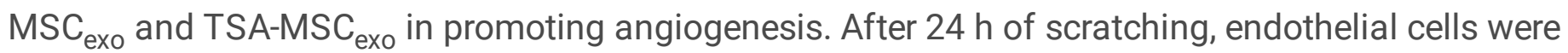
stained for immunofluorescence studies with the endothelial cell markers, CD31 and VEGF. The results showed that VEGF expression increased obviously after treatment with $\mathrm{MSC}_{\text {exo }}$ and TSA-MSC $\mathrm{exo}$ (Supplementary Fig. 1), indicating the ability of $\mathrm{MSC}_{\text {exo }}$ and TSA-MSC $\mathrm{exo}_{\text {on }}$ to enhance proliferation and migration in endothelial cells and promote angiogenesis. TSA-MSC $\mathrm{exo}_{\text {ox }}$ showed a stronger effect than $\mathrm{MSC}_{\text {exo }}$. Thus, $\mathrm{MSC}_{\text {exo }}$ and TSA-MSC $\mathrm{exo}_{\mathrm{o}}$ could inhibit CCR2 activation, reduce monocyte infiltration, and promote angiogenesis after $\mathrm{I} / \mathrm{R}$, with the effect of TSA-MSC $\mathrm{exo}_{\text {ex }}$ being more prominent.

Immunofluorescence co-staining results of CCR2/CD68 and CCR2/a-SMA indicated that CCR2 may have a regulatory effect on monocyte infiltration and angiogenesis.

\section{Overexpression of CCR2 increases monocyte infiltration, inhibits angiogenesis, and weakens the cardioprotective effect of TSA-MSC exo after I/R injury}


To better understand whether CCR2 has a regulatory effect on monocyte infiltration and angiogenesis after myocardial I/R, AAV9 carrying a specific sequence targeting CCR2 was applied to increase CCR2 expression in rat hearts. Eight weeks after AAV-CCR2 $\left(\mathrm{CCR}^{+/+}\right)$or AAV negative control (NC) was injected into the myocardium of rats, western blotting was performed to determine CCR2 expression in the heart tissues. The results showed that the protein expression of CCR2 in the CCR ${ }^{+/+}$group was markedly higher than that in the NC group (Fig. 4A, B), which verified that the myocardial in situ injection of AAVCCR2 successfully upregulated the expression of CCR2 in the rat heart, thereby justifying the development of subsequent experiments. Next, a myocardial I/R model was constructed on the basis of $\mathrm{CCR}^{+/+}$and NC rats. Echocardiographic results showed that the LVEF and LVFS of the CCR2 ${ }^{+/+}$group were not different from those in the NC group. Treatment with TSA-MSC $\mathrm{exo}_{\text {ox }}$ still significantly improved the LVEF and LVFS after I/R (Fig. 4C, D), suggesting that CCR2 overexpression did not aggravate the deterioration of cardiac function. Results from HE and Sirius Red staining showed that collagen deposition and inflammatory cell infiltration in the $\mathrm{CCR} 2^{+/+}$group were obviously increased compared with those in the $\mathrm{NC}$ group. Moreover, in $\mathrm{CCR}^{+/+}$rats, there were no marked differences in inflammatory cell infiltration and collagen deposition between the TSA-MSC $\mathrm{exo}_{\text {and }}$ an Rroups (Fig. 4E, F).

Immunofluorescence co-staining results showed that the expression of both CCR2 and CD68 was significantly increased in the CCR $2^{+/+}$group compared with that in the NC group. In CCR2 ${ }^{+/+}$rats, there

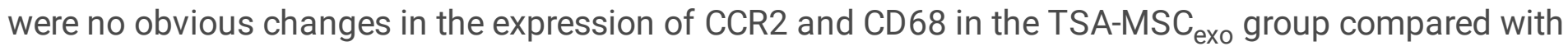
the I/R group (Supplementary Fig. 2A-C). CCR2/a-SMA and CCR2/VEGF immunofluorescence co-staining results indicated that the expression of CCR2 in the $C C R 2^{+/+}$group was obviously higher than that in the NC group, and the expression of a-SMA and VEGF was significantly decreased. In CCR2 ${ }^{+/+}$rats, the expression of CCR2, a-SMA, and VEGF did not change remarkably in the TSA-MSC exo $_{\text {o }}$ group compared with that in the I/R group (Fig. 4G, H). These findings suggested that the overexpression of CCR2 increased monocyte infiltration and inhibited angiogenesis after $\mathrm{I} / \mathrm{R}$ injury such that TSA-MSC exo $_{\text {almost }}$ lost its effect of reducing monocyte infiltration and promoting angiogenesis.

\section{miR-223-5p was a candidate effector for TSA-MSC exo $^{-}$ mediated improvement of cardiac function and myocardial injury}

The biological effects of exosomes depend on the function of miRNAs. Exosomes can exert therapeutic effects on cardiac I/R injury by delivering specific miRNAs to regulate receptor cell function [32-34]. To

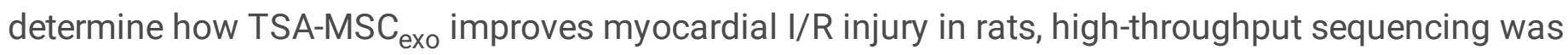
conducted to identify the differentially expressed miRNAs between $M_{S C} C_{\text {exo }}$ and TSA-MSC $C_{\text {exo }}$ (Fig. 5A), in combination with the findings from previous studies that reported that miR-223-5p can regulate inflammation and inhibit I/R injury in mice to promote tissue repair [35-38]. We further verified that the content of miR-223-5p in TSA-MSC $\mathrm{exo}_{\text {ex }}$ was significantly higher than that in $\mathrm{MSC}_{\text {exo }}$ (Fig. 5B). Hence, in subsequent experiments, we explored the role of miR-223-5p in improving I/R injury in rats. The miR-2235p-inhibitory lentivirus (with GFP label) was transfected into MSCs to inhibit miR-223-5p (i223) 
expression, and the lentivirus was used as a vector to transfect into MSCs as an NC. Results from qPCR showed that the lentivirus was successfully transferred into MSCs and remarkably decreased miR-223-5p content in MSCs (Fig. 5C). After successful transfection, the MSCs were treated with TSA and the

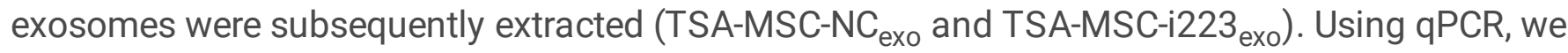
determined that miR-223-5p content in TSA-MSC-i223 $3_{\text {exo }}$ was significantly reduced than that in TSA-MSC$\mathrm{NC}_{\text {exo }}$ (Fig. 5D). After treatment with TSA-MSC-NC exo $_{\text {and TSA-MSC-i223 }}$ exo, the ultrasound results suggested that TSA-MSC-NC $\mathrm{Nxo}_{\text {exo }}$ distinctly increased the LVEF and LVFS of rats compared with those in the I/R group. However, the LVEF and LVFS of rats treated with TSA-MSC-i223 $3_{\text {exo }}$ were not significantly different from those of rats in the I/R group (Fig. 5G, H). TTC staining results suggested that TSA-MSC$\mathrm{NC}_{\text {exo }}$ significantly limited the infarct size of rat heart, and the cardiac infarction area treated with TSAMSC-i223 exo were not significantly different from that of rats in the I/R group (Fig. 5I, J). Results from HE staining showed that inflammatory cell infiltration with TSA-MSC-NC $\mathrm{exo}_{\text {ox }}$ treatment was significantly decreased compared with that in the I/R group. Moreover, inflammatory cell infiltration with TSA-MSCi223 exo treatment was not significantly different from that in the I/R group (Fig. $5 \mathrm{~K}, \mathrm{~L}$ ). The above findings demonstrated that TSA-MSC-i223 $3_{\text {exo }}$ weakens or even abolishes the effect of TSA-MSC-NC exo $_{\text {in }}$ improving cardiac function, limiting infarct size, and reducing inflammatory cell infiltration, suggesting

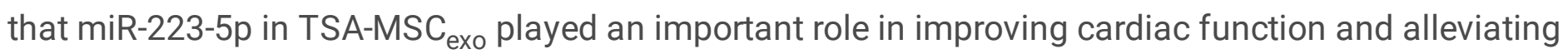
myocardial injury after $\mathrm{I} / \mathrm{R}$ in rats.

\section{miR-223-5p shuttling by TSA-MSC ${ }_{\text {exo }}$ modulated monocyte infiltration and angiogenesis by targeting CCR2}

The interaction between miR-223-5p and the CCR2 gene was predicted using bioinformatics analysis. The results revealed that the free energy of binding between the gene sequence of miR-223-5p derived from humans and the gene sequence of CCR2 derived from different species was lower than $-10 \mathrm{kcal} / \mathrm{mol}$ (Supplementary Fig. 3A), revealing that they could bind stably and interact with each other. Furthermore, in I/R rats injected with PKH26-labeled MSC $_{\text {exo }}$, TSA-MSC-NC exo, $_{\text {, and TSA-MSC-i223 }}$ exo,

immunofluorescence results of the three labeled exosomes and CCR2 indicated that CCR2 expression was lower in the regions where exosomes were present after treatment with $\mathrm{MSC}_{\text {exo }}$ and TSA-MSC-NC $\mathrm{exo}_{\text {, }}$ whereas CCR2 expression in the regions where exosomes were present increased significantly after treatment with TSA-MSC-i223 $3_{\text {exo }}$ (Fig. 6A-C), suggesting the key role of miR-223-5p in inhibiting CCR2 activation. Furthermore, immunofluorescence results of CCR2 and CD68 co-staining showed that the positive expression of CCR2 and CD68 in the myocardium of rats treated with TSA-MSC-NC exo $_{\text {was }}$ distinctly decreased compared with that of rats in the I/R group, and the positive expression of CCR2 and CD68 in the myocardium of rats treated with TSA-MSC-i223 $3_{\text {exo }}$ did not change significantly compared with those in the I/R group (Supplementary Fig. 3B-D). Immunofluorescence results of CCR2/a-SMA and CCR2/VEGF co-staining demonstrated that the positive expression of CCR2 in the myocardium of rats treated with TSA-MSC-NC $\mathrm{Nexo}_{\text {o }}$ was significantly reduced compared with that in the I/R group, and the positive expression of a-SMA and VEGF was significantly increased. However, the positive expression of 
CCR2, a-SMA, and VEGF in the myocardium of rats treated with TSA-MSC-i223 $3_{\text {exo }}$ did not change significantly compared with that in the I/R group (Fig. 6D-I). Results from Sirius Red staining showed that

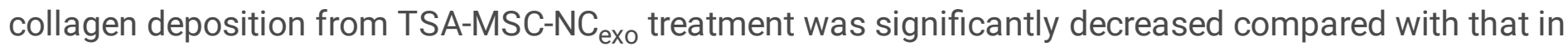
the I/R group. Moreover, collagen deposition and from TSA-MSC-i223 exo treatment was not significantly different from that in the l/R group (Fig. $6 \mathrm{~J}, \mathrm{~K}$ ). These results indicated that diminishing miR-223-5p weakens or even almost eliminates the regulatory effect of TSA-MSC $\mathrm{Cxo}_{\text {ox }}$ on monocyte infiltration and angiogenesis after $\mathrm{I} / \mathrm{R}$ injury, suggesting that miR-223-5p encapsulated in TSA-MSC $\mathrm{exo}_{\text {o }}$ modulated monocyte infiltration and angiogenesis after $\mathrm{I} / \mathrm{R}$ by inhibiting the activation of CCR2.

\section{Discussion}

In recent years, the development of stem cell regenerative medicine has brought new hope for the therapy of cardiovascular diseases. Several studies have shown that stem cells secrete paracrine factors, including exosomes, to exert cardioprotective effects [33]. MSC-derived exosomes can promote the repair of damaged myocardium and improve cardiac function after myocardial injury, suggesting the potential of exosome-based approaches as novel cell-free therapy in cardiac repair [34, 39, 40]. In this study, we

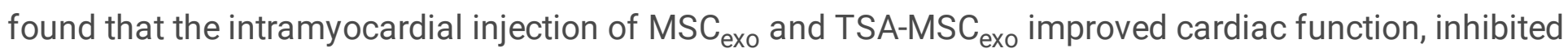
CCR2 activation, reduced monocyte infiltration, and promoted angiogenesis after myocardial I/R injury. In addition, CCR2 was found to have a regulatory effect on monocyte infiltration and angiogenesis after I/R. It was worth noting that TSA-MSC $\mathrm{exo}_{\text {o }}$ was more effective in improving cardiac function and myocardial

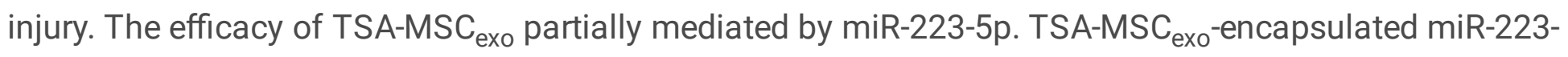
$5 p$ was transported to the injured heart tissue to inhibit CCR2 activation, thereby reducing monocyte infiltration and promoting angiogenesis to exert a cardioprotective effect (Fig. 7). To our knowledge, this is the first study to determine the cardioprotective effects of TSA-pretreated MSC-derived exosomes.

Therapy with stem cell-derived exosomes shows low immunogenicity and low carcinogenic risk and similar effects as those observed with stem cell transplantation in the treatment of myocardial I/R injury. In recent years, the modification of stem cell-derived exosomes has shown excellent outcomes in repairing myocardial injury. Studies have confirmed that the expression of anti-apoptotic miRNAs in exosomes derived from GATA4-overexpressing MSCs increases and, thus, enhances cardioprotection in $\mathrm{MI}$ rats [41]. Another study confirmed that the Akt-modified MSCs-derived exosomes enhanced angiogenesis by activating growth factor $D$ [42]. Although these modifications may increase the expression of cytokines or miRNA and enhance the therapeutic activity of exosomes, these outcomes are currently difficult to achieve in clinical practice. Pharmacological studies have shown that TSA can protect the heart from ischemic damage and may be a promising therapeutic agent for the treatment of myocardial I/R injury and arrhythmia; however, the poor bioavailability of TSA limits its efficacy [43]. In this study, the development of an alternative therapy for TSA-pretreated MSC-derived exosomes not only improved the shortcomings of TSA but also increased the therapeutic activity of exosomes. Our findings

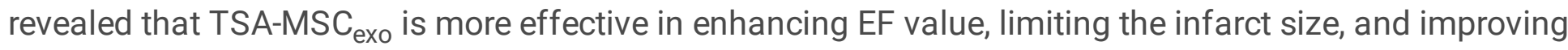
myocardial damage after I/R compared with that observed using $\mathrm{MSC}_{\text {exo }}$ treatment. Therefore, our 
findings may provide a prospective strategy for the treatment of cardiovascular diseases and create the possibility for the use of TSA in a clinical setting.

Proinflammatory monocytes are recruited to the damaged myocardium after myocardial I/R to induce an inflammatory response, which is accompanied by the subsequent release of proinflammatory mediators and enhanced protease activity to exacerbate the inflammatory response. The continuous development of inflammation hinders myocardial tissue repair [39]. However, stimulating the growth of small blood vessels and the formation of collateral circulation in the ischemic regions of the myocardium is beneficial for tissue repair after myocardial injury [44]. Recent studies have demonstrated that exosomes isolated from stem cells, such as MSCs and cardiomyocyte-derived cells (CDCs), have the ability to enhance angiogenesis and reduce inflammation. Consistently, our results confirmed that $\mathrm{MSC}_{\text {exo }}$ had the ability to inhibit the infiltration of monocytes and promote angiogenesis. Furthermore, TSA-MSC $\mathrm{exo}_{\mathrm{s}}$ showed greater potential with respect to these aspects. Studies show that CCR2 activation leads to the recruitment of monocytes to infiltrate the infarct area and cause abnormal remodeling of coronary blood vessels, thereby exacerbating myocardial I/R injury $[14,15]$. The results from our study indicated that TSA-MSC exo significantly reduced CCR2 expression. Based on these findings, we injected AAV9-CCR2 into the rat heart to increase CCR2 expression and successfully established the myocardial I/R model. We discovered that the overexpression of CCR2 promoted the infiltration of monocytes into the injured heart and interfered with angiogenesis after $\mathrm{I} / \mathrm{R}$, thus exacerbating myocardial injury and weakening the cardioprotective effect of TSA-MSC exo $_{\text {. }}$.

Increasing evidence reveals that the biological effects of exosomes depend on the function of miRNAs, which means that exosomes regulate the function of cellular receptors and interfere with the pathophysiological processes of the body by delivering specific miRNAs [32, 33]. Certain exosomal miRNAs, such as miR-21, miR-223, miR-146a, and miR-181b, derived from stem cells have important immunomodulatory properties [27, 45-47]. miR-223-5p is an effective negative regulator of cardiac inflammation, which inhibits monocyte infiltration and prevents polarization of M1 macrophages by interfering with one or more targets including NF-KB, IKKa, and STAT3, thereby playing a role in cardiovascular protection $[35,48,49]$. To date, only a few studies have explored the relationship between miR-223-5p and myocardial I/R injury. Based on the findings from our sequencing data, supported by the previous literature, we identified miR-223-5p as the preferred research object. In this study, the expression of miR-223-5p in TSA-pretreated MSC-derived exosomes was remarkably higher in TSA-MSC $\mathrm{exo}_{\text {ox }}$ than in

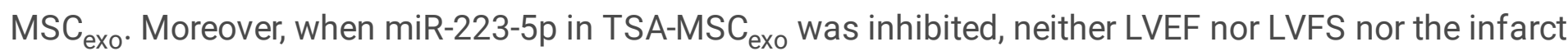
size of rats with I/R injury were significantly recovered. Our findings prove, for the first time, that the decline of miR-223-5p expression in TSA-MSC $\mathrm{C}_{\text {exo }}$ greatly weakened the regulatory effects of TSA-MSC $\mathrm{exo}_{\text {o }}$ on the cardiac function in rats after I/R injury. Furthermore, when the expression of miR-223-5p in TSA$\mathrm{MSC}_{\text {exo }}$ was suppressed, CCR2 expression was not significantly downregulated. Thus, bioinformatics analysis was used to identify the interaction between miR-223-5p and CCR2. We found that when the miR-223-5p level in TSA-MSC exo $_{\text {o }}$ decreased, its efficacy in inhibiting CCR2 activation was partially reversed and was accompanied by an increase in monocyte infiltration and inhibition of angiogenesis. To 
the best of our knowledge, the relationship between miR-223-5p and CCR2 has not been reported previously. Our findings confirmed that TSA-MSC ${ }_{\text {exo }}$ inhibited CCR2 by transporting miR-223-5p into the heart to inhibit monocyte infiltration and promote angiogenesis, thus alleviating I/R injury.

Despite conclusive evidence indicating that miR-223-5p in exosomes is essential for cardioprotective effects after I/R, many other components in exosomes also have biological activity and can synergistically exhibit overall functional advantages. Therefore, we cannot ignore the contribution of other exosomal contents. Although there is evidence that CCR2 may be the downstream target protein of exosomal miR-223-5p, it is clear that exosomes and their miRNAs have effects on proteins. Thus, the possibility of their synergistic effects cannot be ruled out, and merely targeting CCR2 as a research object

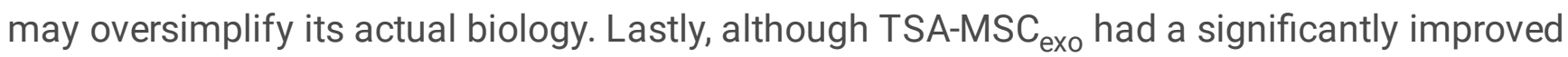
therapeutic affection compared with $\mathrm{MSC}_{\mathrm{exo}}$, further preclinical studies are needed to ensure its efficacy and safety, prior to this strategy being developed into a potential cell-free therapy for myocardial repair.

\section{Conclusion}

Overall, we found that TSA-MSC exo $_{0}$ could ameliorate cardiac function, limit infarct size, inhibit the activation of CCR2, reduce monocyte infiltration, and promote angiogenesis to attenuate myocardial I/R

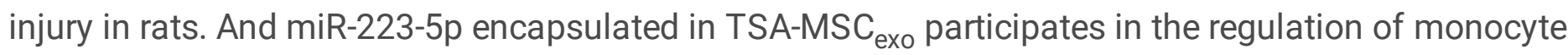
infiltration and angiogenesis by targeting CCR2. The therapy of TSA-pretreated MSC-derived exosomes provides a prospective strategy in a clinical setting in the management of cardiovascular diseases.

\section{Abbreviations}

AAV, adeno-associated virus; CDCs, cardiomyocyte-derived cells; CHD, Coronary heart disease; DAMPs, damage-associated molecular patterns; DLS, dynamic light scattering; DMEM, Dulbecco's modified Eagle's medium; ECM, endothelial cell medium; FBS, fetal bovine serum; HE, hematoxylin/eosin; HRP, horseradish peroxidase; HUMSCs, human umbilical mesenchymal stem cells; HUVECs, human umbilical vein endothelial cells; I/R, ischemia-reperfusion; LVEF, left ventricular ejection fraction; LVFS, left ventricular fraction shortening; MHCll, major histocompatibility complex class II; miRNA, microRNA; MSCM, mesenchymal stem cell medium; MSCs, Mesenchymal stem cells; NC, negative control; NGS, Next-generation sequencing; PBS, phosphate-buffered saline; PCl, percutaneous coronary intervention; PV, pressure-volume; qPCR, quantitative polymerase chain reaction; TEM, transmission electron microscopy; TSA, tanshinone IIA; TTC, 2,3,5-Triphenyltetrazolium chloride; TUNEL, terminal deoxynucleotidyl transferase dUTP nick end labeling; VEGF, vascular endothelial growth factor.

\section{Declarations}

\section{Acknowledgements}




\section{Authors' contribution}

SL, LL, CRM and GWF participated in the conceptualization and design of the study. RG, WLC, ZHL and JZ participated in the image analysis. QQL and YTH participated in the statistical analysis of the data. SL and LL participated in drafting the manuscript. All authors read and approved the final manuscript.

\section{Funding}

This work was supported by the Tianjin Outstanding Youth Science Foundation (17JCJQJC46200) and the National Natural Science Foundation of China (81774050).

\section{Availability of date and materials}

The datasets used during the current study are available from the corresponding authors on reasonable request.

\section{Ethics approval and consent to participate}

All animal procedures were approved by the Tianjin University of Traditional Chinese Medicine Animal Research Committee (TCM-LAEC2014005) and performed in accordance with Directive 2010/63/EU of the European Parliament.

\section{Consent for publication}

Not applicable.

\section{Competing interests}

The authors declare that they have no competing interests.

\section{References}

1. Correction to: Heart Disease and Stroke Statistics-2019 Update: A Report From the American Heart Association. Circulation. 2020;141(2):e33. 
2. Magro M, Garg S, Serruys PW. Revascularization treatment of stable coronary artery disease. Expert Opin Pharmacother. 2011;12(2):195-212.

3. Frohlich GM, Meier P, White SK, Yellon DM, Hausenloy DJ. Myocardial reperfusion injury: looking beyond primary PCI. Eur Heart J. 2013;34(23):1714-22.

4. Panizzi P, Swirski FK, Figueiredo JL, Waterman P, Sosnovik DE, Aikawa E, et al. Impaired infarct healing in atherosclerotic mice with Ly-6C(hi) monocytosis. J Am Coll Cardiol. 2010;55(15):1629-38.

5. van der Laan AM, Piek JJ, van Royen $\mathrm{N}$. Targeting angiogenesis to restore the microcirculation after reperfused MI. Nat Rev Cardiol. 2009;6(8):515-23.

6. Shah AM, Mann DL. In search of new therapeutic targets and strategies for heart failure: recent advances in basic science. Lancet. 2011;378(9792):704-12.

7. Frangogiannis NG. The mechanistic basis of infarct healing. Antioxid Redox Signal. 2006;8(1112):1907-39.

8. Bajpai G, Bredemeyer A, Li W, Zaitsev K, Koenig AL, Lokshina I, et al. Tissue Resident CCR2- and CCR2+ Cardiac Macrophages Differentially Orchestrate Monocyte Recruitment and Fate Specification Following Myocardial Injury. Circ Res. 2019;124(2):263-78.

9. Frodermann V, Nahrendorf M. Macrophages and Cardiovascular Health. Physiol Rev. 2018;98(4):2523-69.

10. Williams JW, Giannarelli C, Rahman A, Randolph GJ, Kovacic JC. Macrophage Biology, Classification, and Phenotype in Cardiovascular Disease: JACC Macrophage in CVD Series (Part 1). J Am Coll Cardiol. 2018;72(18):2166-80.

11. Fayad ZA, Swirski FK, Calcagno C, Robbins CS, Mulder W, Kovacic JC. Monocyte and Macrophage Dynamics in the Cardiovascular System: JACC Macrophage in CVD Series (Part 3). J Am Coll Cardiol. 2018;72(18):2198-212.

12. Lavine KJ, Pinto AR, Epelman S, Kopecky BJ, Clemente-Casares X, Godwin J, et al. The Macrophage in Cardiac Homeostasis and Disease: JACC Macrophage in CVD Series (Part 4). J Am Coll Cardiol. 2018;72(18):2213-30.

13. Ong SB, Hernandez-Resendiz S, Crespo-Avilan GE, Mukhametshina RT, Kwek XY, Cabrera-Fuentes HA, et al. Inflammation following acute myocardial infarction: Multiple players, dynamic roles, and novel therapeutic opportunities. Pharmacol Ther. 2018;186:73-87.

14. Dutta P, Sager HB, Stengel KR, Naxerova K, Courties G, Saez B, et al. Myocardial Infarction Activates CCR2(+) Hematopoietic Stem and Progenitor Cells. Cell Stem Cell. 2015;16(5):477-87.

15. Leid J, Carrelha J, Boukarabila H, Epelman S, Jacobsen SE, Lavine KJ. Primitive Embryonic Macrophages are Required for Coronary Development and Maturation. Circ Res. 2016;118(10):1498511.

16. Lu W, Xie Z, Tang Y, Bai L, Yao Y, Fu C, et al. Photoluminescent Mesoporous Silicon Nanoparticles with siCCR2 Improve the Effects of Mesenchymal Stromal Cell Transplantation after Acute Myocardial Infarction. Theranostics. 2015;5(10):1068-82. 
17. Huang P, Wang L, Li Q, Tian X, Xu J, Xu J, et al. Atorvastatin enhances the therapeutic efficacy of mesenchymal stem cells-derived exosomes in acute myocardial infarction via up-regulating long non-coding RNA H19. Cardiovasc Res. 2020;116(2):353-67.

18. Ruan XF, Ju CW, Shen Y, Liu YT, Kim IM, Yu H, et al. Suxiao Jiuxin pill promotes exosome secretion from mouse cardiac mesenchymal stem cells in vitro. Acta Pharmacol Sin. 2018;39(4):569-78.

19. Ruan XF, Li YJ, Ju CW, Shen Y, Lei W, Chen C, et al. Exosomes from Suxiao Jiuxin pill-treated cardiac mesenchymal stem cells decrease H3K27 demethylase UTX expression in mouse cardiomyocytes in vitro. Acta Pharmacol Sin. 2018;39(4):579-86.

20. Hu H, Zhai C, Qian G, Gu A, Liu J, Ying F, et al. Protective effects of tanshinone IIA on myocardial ischemia reperfusion injury by reducing oxidative stress, HMGB1 expression, and inflammatory reaction. Pharm Biol. 2015;53(12):1752-8.

21. Li Q, Shen L, Wang Z, Jiang HP, Liu LX. Tanshinone IIA protects against myocardial ischemia reperfusion injury by activating the PI3K/Akt/mTOR signaling pathway. Biomed Pharmacother. 2016;84:106-14.

22. Zhang MQ, Zheng YL, Chen $H$, Tu JF, Shen Y, Guo JP, et al. Sodium tanshinone IIA sulfonate protects rat myocardium against ischemia-reperfusion injury via activation of $\mathrm{PI} / \mathrm{K} / \mathrm{Akt} / \mathrm{FOXO} \mathrm{A} / \mathrm{Bim}$ pathway. Acta Pharmacol Sin. 2013;34(11):1386-96.

23. Zhang Y, Wei L, Sun D, Cao F, Gao H, Zhao L, et al. Tanshinone IIA pretreatment protects myocardium against ischaemia/reperfusion injury through the phosphatidylinositol 3-kinase/Akt-dependent pathway in diabetic rats. Diabetes Obes Metab. 2010;12(4):316-22.

24. Wei B, Li WW, Ji J, Hu QH, Ji H. The cardioprotective effect of sodium tanshinone IIA sulfonate and the optimizing of therapeutic time window in myocardial ischemia/reperfusion injury in rats. Atherosclerosis. 2014;235(2):318-27.

25. Barile L, Cervio E, Lionetti V, Milano G, Ciullo A, Biemmi V, et al. Cardioprotection by cardiac progenitor cell-secreted exosomes: role of pregnancy-associated plasma protein-A. Cardiovasc Res. 2018;114(7):992-1005.

26. Thery C, Amigorena S, Raposo G, Clayton A. Isolation and characterization of exosomes from cell culture supernatants and biological fluids. Curr Protoc Cell Biol. 2006;Chapter 3:Unit 322.

27. de Couto G, Gallet R, Cambier L, Jaghatspanyan E, Makkar N, Dawkins JF, et al. Exosomal MicroRNA Transfer Into Macrophages Mediates Cellular Postconditioning. Circulation. 2017;136(2):200-14.

28. Li L, Ni J, Li M, Chen J, Han L, Zhu Y, et al. Ginsenoside Rg3 micelles mitigate doxorubicin-induced cardiotoxicity and enhance its anticancer efficacy. Drug Deliv. 2017;24(1):1617-30.

29. Frantz S, Nahrendorf M. Cardiac macrophages and their role in ischaemic heart disease. Cardiovasc Res. 2014;102(2):240-8.

30. Gordon S, Taylor PR. Monocyte and macrophage heterogeneity. Nat Rev Immunol. 2005;5(12):95364.

31. Nahrendorf M, Pittet MJ, Swirski FK. Monocytes: protagonists of infarct inflammation and repair after myocardial infarction. Circulation. 2010;121(22):2437-45. 
32. Kalluri R, LeBleu VS. The biology, function, and biomedical applications of exosomes. Science. 2020;367(6478).

33. Mathieu M, Martin-Jaular L, Lavieu G, Thery C. Specificities of secretion and uptake of exosomes and other extracellular vesicles for cell-to-cell communication. Nat Cell Biol. 2019;21(1):9-17.

34. Xu R, Zhang F, Chai R, Zhou W, Hu M, Liu B, et al. Exosomes derived from pro-inflammatory bone marrow-derived mesenchymal stem cells reduce inflammation and myocardial injury via mediating macrophage polarization. J Cell Mol Med. 2019;23(11):7617-31.

35. Qin D, Wang X, Li Y, Yang L, Wang R, Peng J, et al. MicroRNA-223-5p and -3p Cooperatively Suppress Necroptosis in Ischemic/Reperfused Hearts. J Biol Chem. 2016;291(38):20247-59.

36. Gou W, Zhang Z, Yang C, Li Y. MiR-223/Pknox1 axis protects mice from CVB3-induced viral myocarditis by modulating macrophage polarization. Exp Cell Res. 2018;366(1):41-8.

37. Valmiki S, Ahuja V, Puri N, Paul J. miR-125b and miR-223 Contribute to Inflammation by Targeting the Key Molecules of NFkappaB Pathway. Front Med (Lausanne). 2019;6:313.

38. Chen L, Hou X, Zhang M, Zheng Y, Zheng X, Yang Q, et al. MicroRNA-223-3p modulates dendritic cell function and ameliorates experimental autoimmune myocarditis by targeting the NLRP3 inflammasome. Mol Immunol. 2020;117:73-83.

39. Liang X, Zhang L, Wang S, Han Q, Zhao RC. Exosomes secreted by mesenchymal stem cells promote endothelial cell angiogenesis by transferring miR-125a. J Cell Sci. 2016;129(11):2182-9.

40. Ma T, Chen Y, Chen Y, Meng Q, Sun J, Shao L, et al. MicroRNA-132, Delivered by Mesenchymal Stem Cell-Derived Exosomes, Promote Angiogenesis in Myocardial Infarction. Stem Cells Int. 2018;2018:3290372.

41. Yu B, Kim HW, Gong M, Wang J, Millard RW, Wang Y, et al. Exosomes secreted from GATA-4 overexpressing mesenchymal stem cells serve as a reservoir of anti-apoptotic microRNAs for cardioprotection. Int J Cardiol. 2015;182:349-60.

42. Ma J, Zhao Y, Sun L, Sun X, Zhao X, Sun X, et al. Exosomes Derived from Akt-Modified Human Umbilical Cord Mesenchymal Stem Cells Improve Cardiac Regeneration and Promote Angiogenesis via Activating Platelet-Derived Growth Factor D. Stem Cells Transl Med. 2017;6(1):51-9.

43. Li ZM, Xu SW, Liu PQ. Salvia miltiorrhizaBurge (Danshen): a golden herbal medicine in cardiovascular therapeutics. Acta Pharmacol Sin. 2018;39(5):802-24.

44. Sun $X H$, Wang $X$, Zhang $Y$, Hui J. Exosomes of bone-marrow stromal cells inhibit cardiomyocyte apoptosis under ischemic and hypoxic conditions via miR-486-5p targeting the PTEN/PI3K/AKT signaling pathway. Thromb Res. 2019;177:23-32.

45. Wang X, Gu H, Qin D, Yang L, Huang W, Essandoh K, et al. Exosomal miR-223 Contributes to Mesenchymal Stem Cell-Elicited Cardioprotection in Polymicrobial Sepsis. Sci Rep. 2015;5:13721.

46. Xiao J, Pan Y, Li XH, Yang XY, Feng YL, Tan HH, et al. Cardiac progenitor cell-derived exosomes prevent cardiomyocytes apoptosis through exosomal miR-21 by targeting PDCD4. Cell Death Dis. 2016;7(6):e2277. 
47. Song Y, Dou H, Li X, Zhao X, Li Y, Liu D, et al. Exosomal miR-146a Contributes to the Enhanced Therapeutic Efficacy of Interleukin-1 beta-Primed Mesenchymal Stem Cells Against Sepsis. Stem Cells. 2017;35(5):1208-21.

48. Wang K, Long B, Liu F, Wang JX, Liu CY, Zhao B, et al. A circular RNA protects the heart from pathological hypertrophy and heart failure by targeting miR-223. Eur Heart J. 2016;37(33):2602-11.

49. Shi L, Kojonazarov B, Elgheznawy A, Popp R, Dahal BK, Bohm M, et al. miR-223-IGF-IR signalling in hypoxia- and load-induced right-ventricular failure: a novel therapeutic approach. Cardiovasc Res. 2016;111(3):184-93.

\section{Figures}




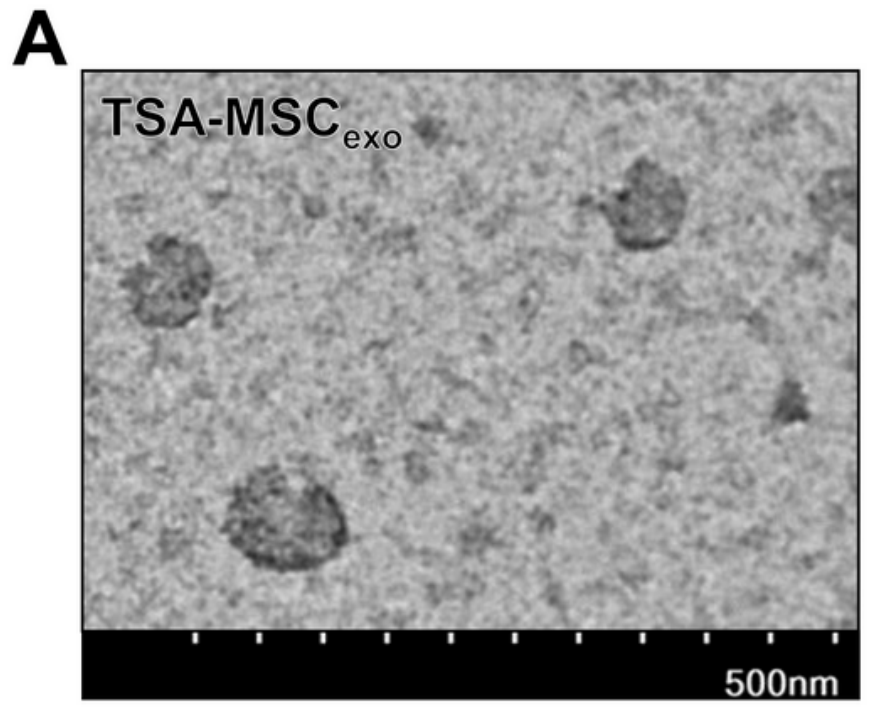

B

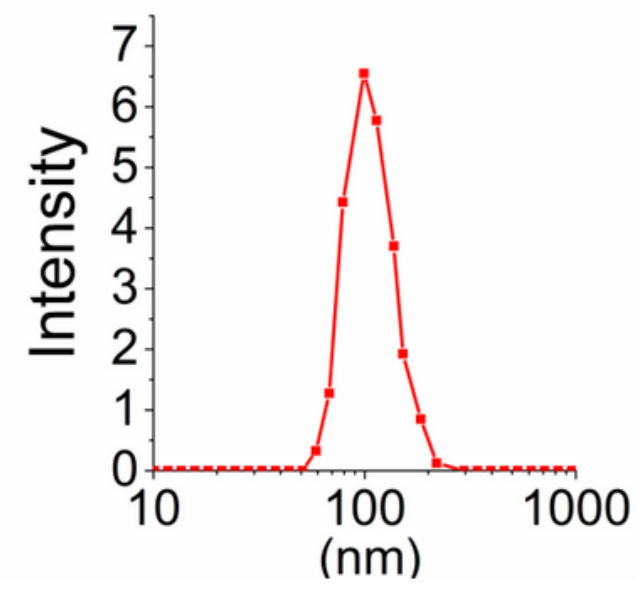

C

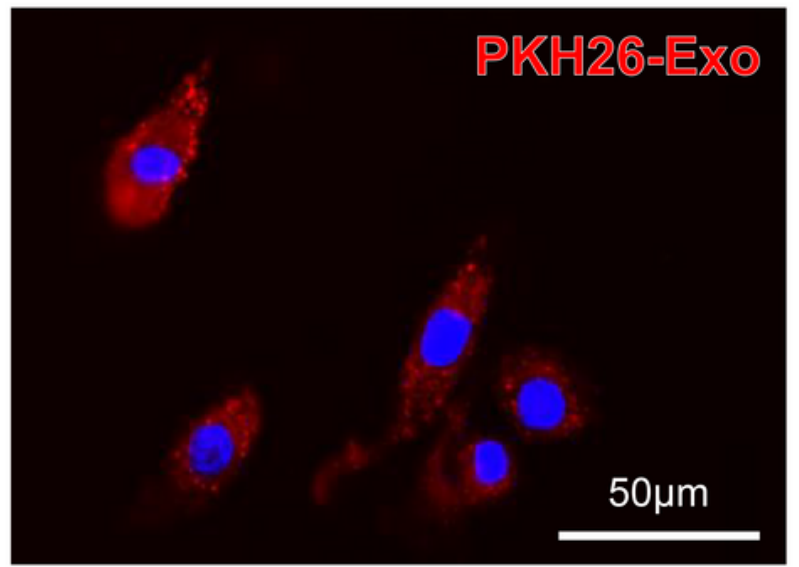

D

MSC $_{\text {exo }}$ TSA-MSC

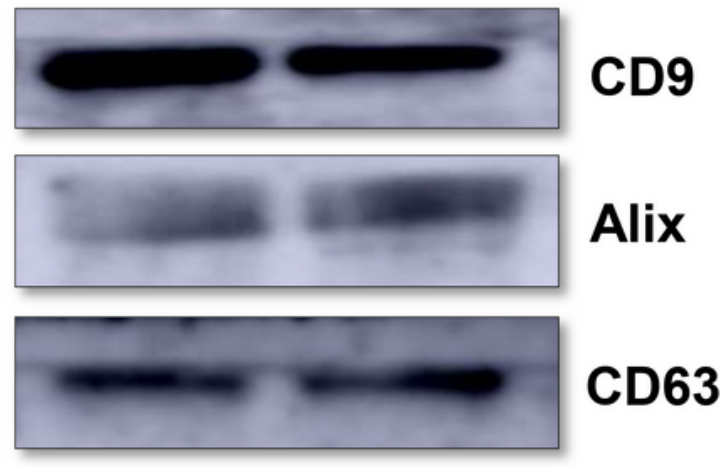

$\mathbf{E}$

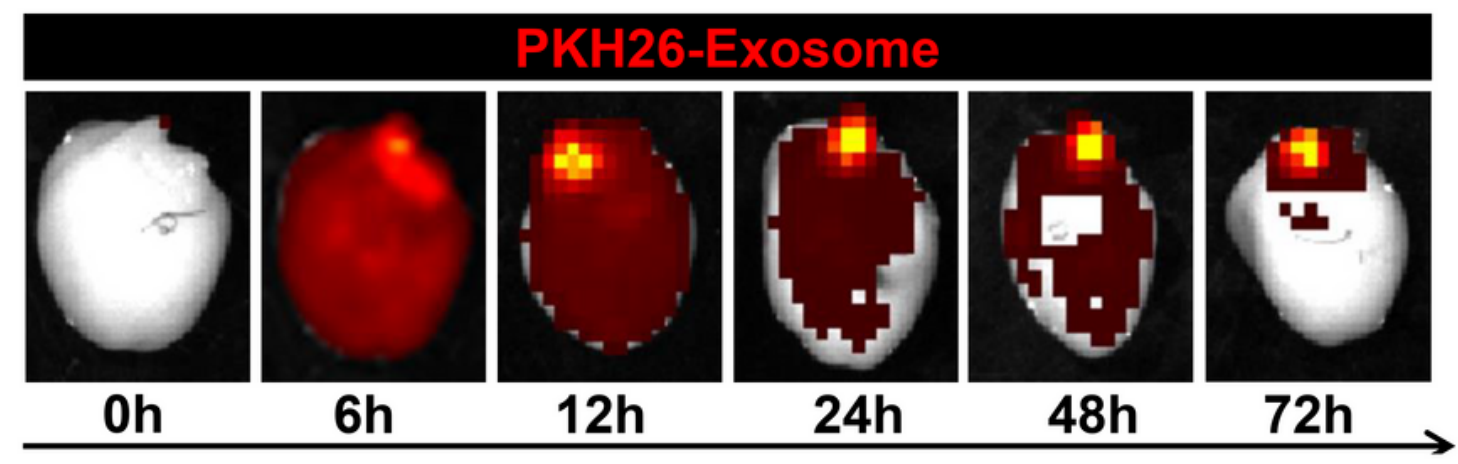

Figure 1

Characterization and internalization of exosomes. A TEM showing TSA-MSCexo as round vesicles with a lipid bilayer (scale bar $=500 \mathrm{~nm}$ ). B DLS analysis to determine the hydrodynamic diameter of exosomes. C Representative immunofluorescence images showing the internalization of exosomes in $\mathrm{H} 9 \mathrm{C} 2$ cells $($ scale bar $=50 \mu \mathrm{m})$. D Marker proteins of exosomes were detected using western blotting. E Near-infrared 
detection of rat cardiomyocytes showing the internalization of exosomes and the residence time of exosomes in the body. Data are presented from three independent experiments.

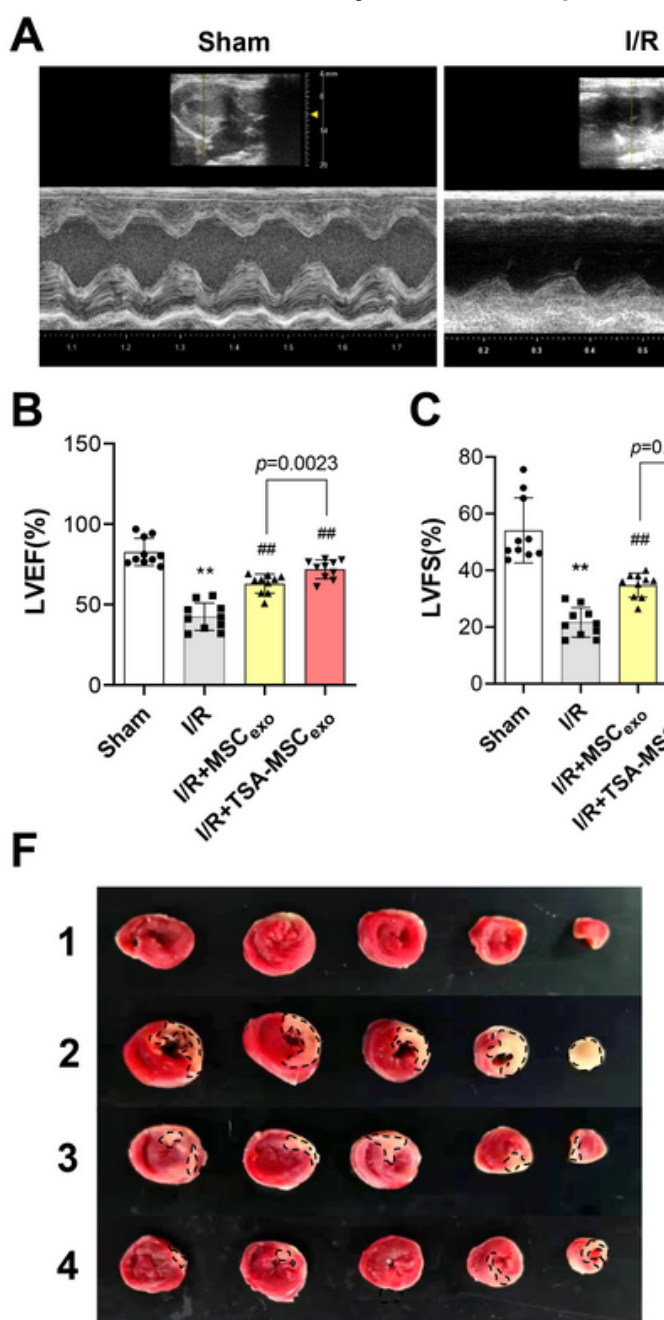

1: Sham 2: I/R 3: I/R + MSC exo $_{\text {in }} 4: I / R+T S A-M S C_{\text {exo }}$
I/R

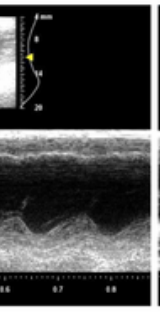

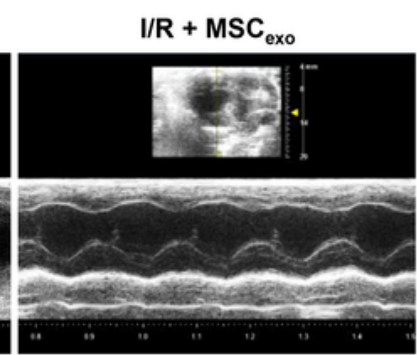

D

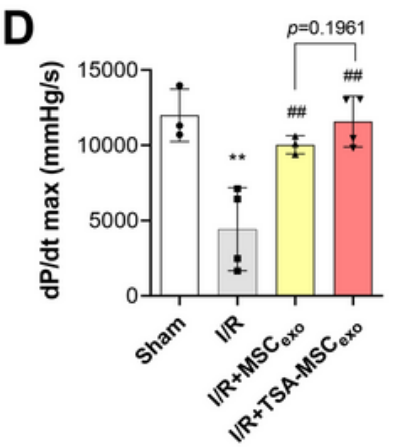

E

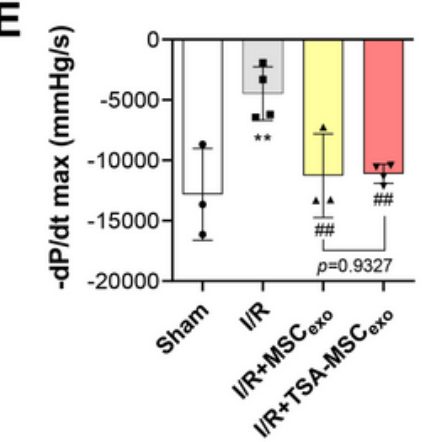

$\mathbf{H}$
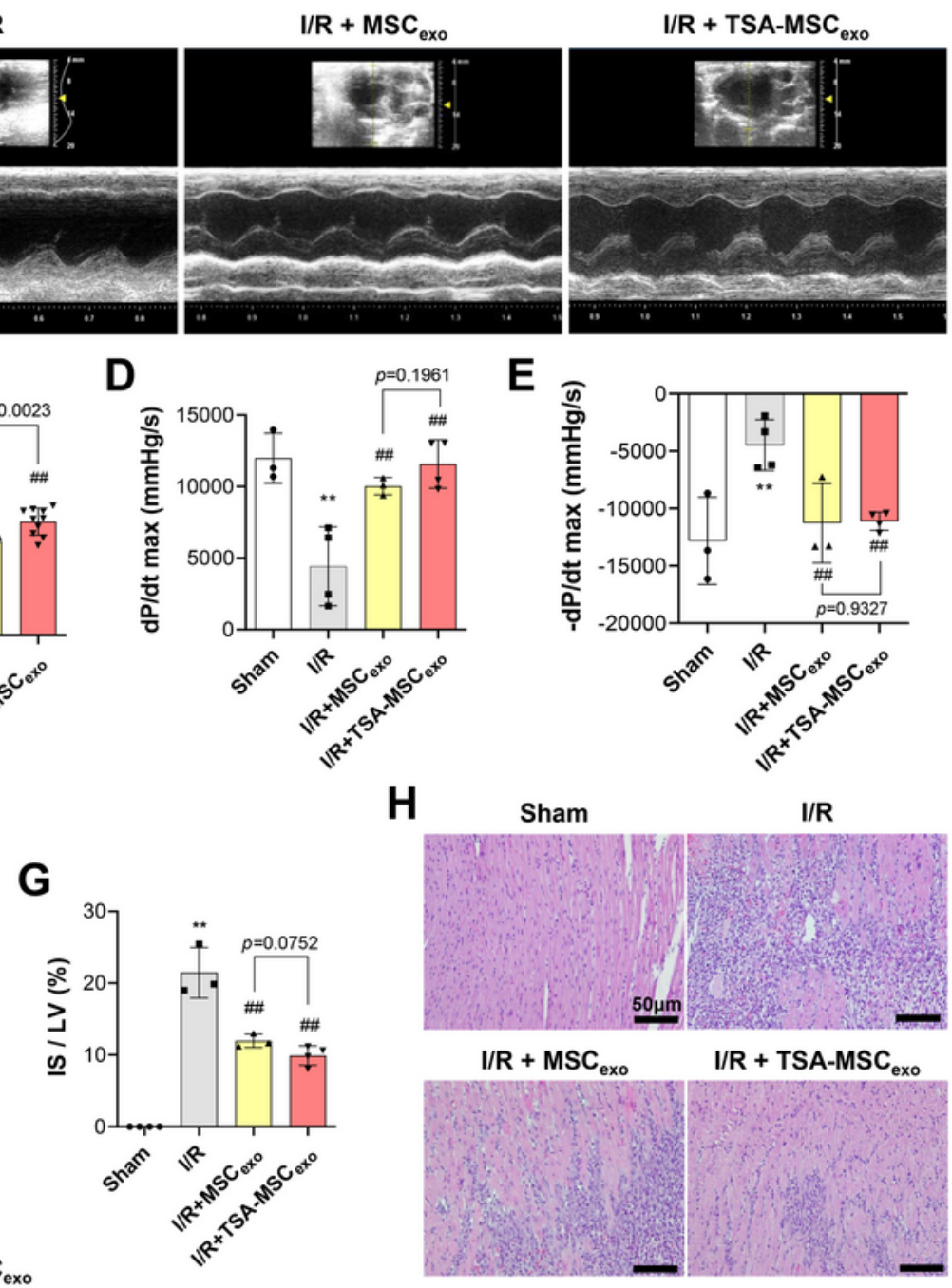

$\mathbf{J}$
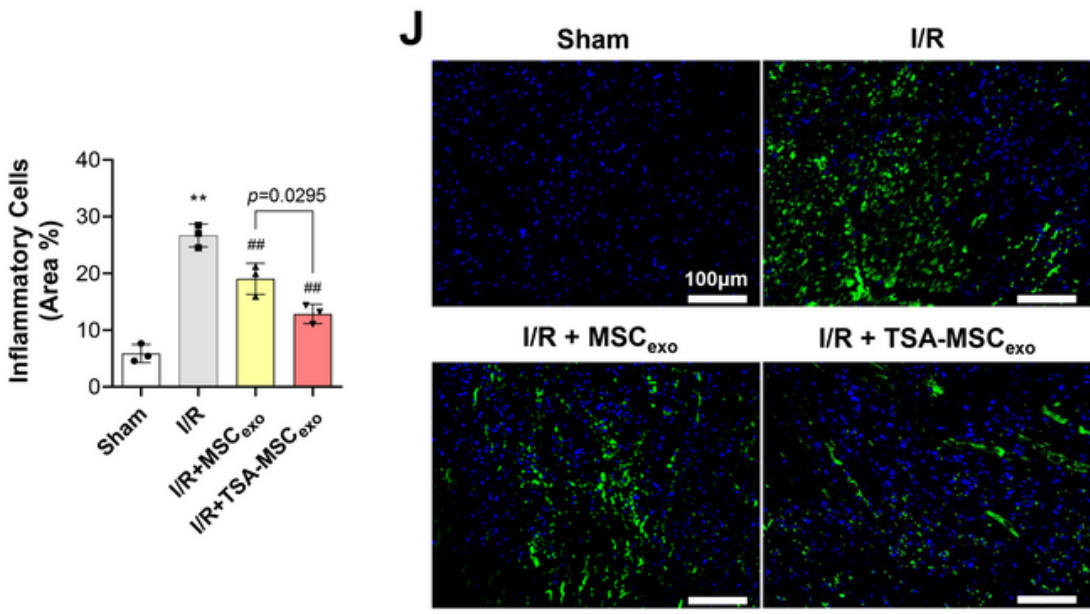

K

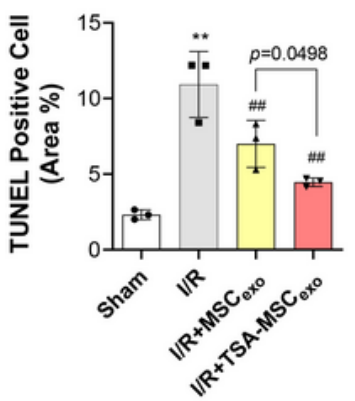

\section{Figure 2}

Improvement of TSA-MSCexo on cardiac function and myocardial injury in rats with I/R. A Representative images of M-mode two-dimensional ultrasound 3 days after reperfusion. B, C Quantitative analysis of LVEF and LVFS 3 days after reperfusion $(n=10)$. D, E Quantitative analysis of $\mathrm{dP} / \mathrm{dt}$ max and $\mathrm{dP} / \mathrm{dt}$ min 
3 days after reperfusion $(n=3)$. F Representative images of TTC staining of heart sections 3 days after reperfusion. G Quantification of infarct size percentage $(n=3)$. H, I Representative images and quantification of HE staining of a cross-section of the heart 3 days after reperfusion (scale bar $=50 \mu \mathrm{m}$ ). $\mathrm{J}, \mathrm{K}$ Representative images of TUNELstaining of a cross-section of the heart 3 days after reperfusion $($ scale bar $=100 \mu \mathrm{m})$. Data are expressed as mean \pm SD. $* *, p<0.01$ compared with the sham group; \#\#, $\mathrm{p}<0.01$ compared with the I/R group.
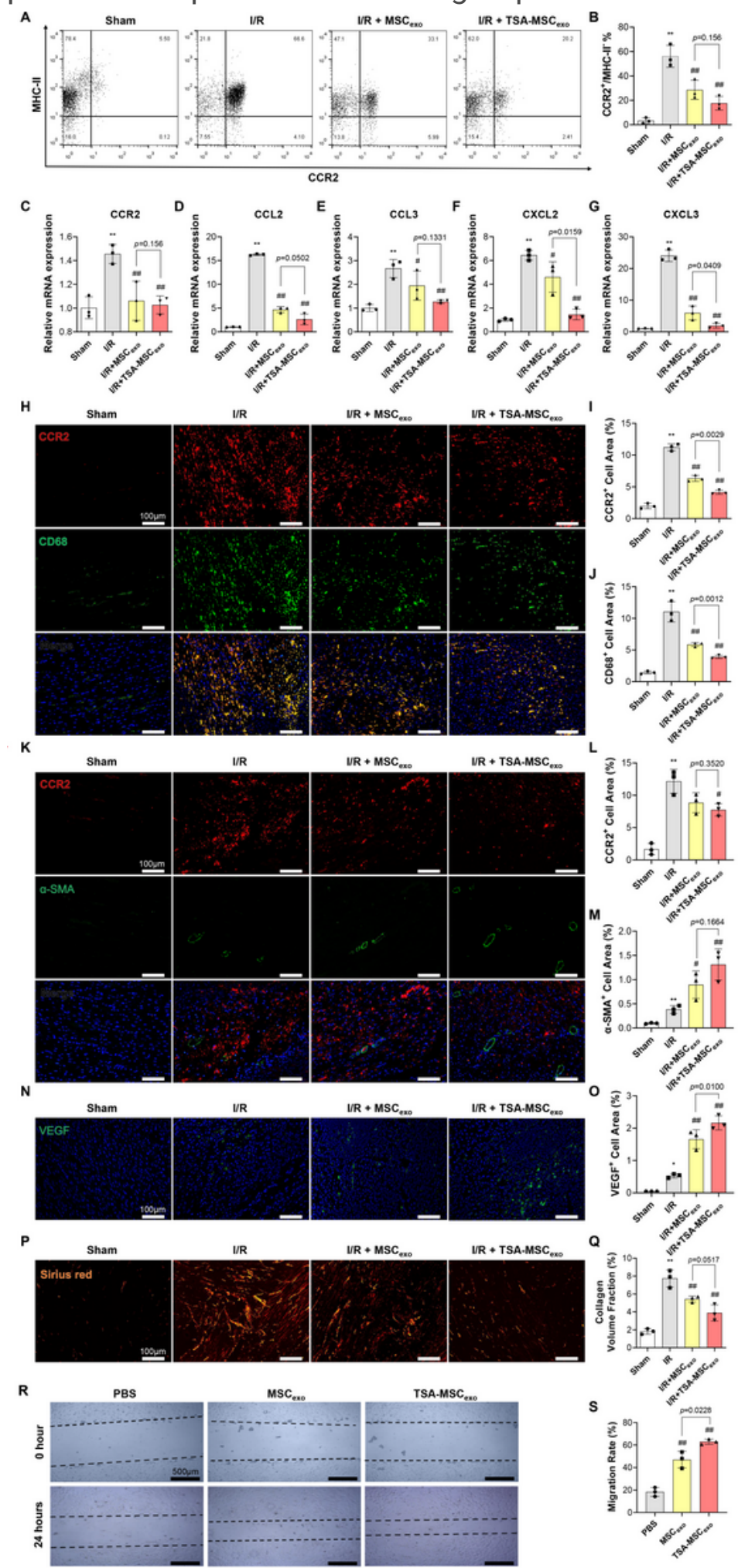

MSC $_{\text {eet }}$

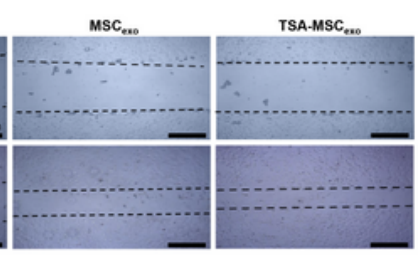

Figure 3 
Effect of TSA-MSCexo on CCR2 activation, monocyte infiltration, and angiogenesis after myocardial I/R. A Representative image of CCR2 and MHC flow cytometry in myocardial tissues. B Quantification of the percentage of CCR2+MHCII- cells in myocardial tissue $(n=3)$. C CCR2 gene expression in myocardial tissue $(n=3)$. D-G Relative mRNA expression of CCL2, CCL3, CXCL2, and CXCL3 in myocardial tissue ( $n=$ 3). Standardization of the expression of the target gene relative to GADPH to express it as a relative change. H-J Representative images and quantification of CCR2 and CD68 immunofluorescence of cardiac tissue sections (scale bar $=100 \mu \mathrm{m}$ ). K-M Representative images and quantification of CCR2 and a-SMA immunofluorescence of cardiac tissue sections (scale bar $=100 \mu \mathrm{m}$ ). N, O Representative images and quantification of VEGF immunofluorescence of cardiac tissue sections (scale bar $=100 \mu \mathrm{m}$ ). P, Q Representative images and quantification of Sirius Red staining of a cross-section of the heart 3 days after reperfusion (scale bar $=100 \mu \mathrm{m}$ ). R, S Representative images of endothelial cells from scratches at 0 and $24 \mathrm{~h}$ and quantification of endothelial cells migration rate (scale bar $=500 \mu \mathrm{m}$ ). Data are expressed as mean $\pm S D$. $*, p<0.05, * *, p<0.01$ compared with the sham group; $\#, p<0.05, \# \#, p<0.01$ compared with the I/R group. 


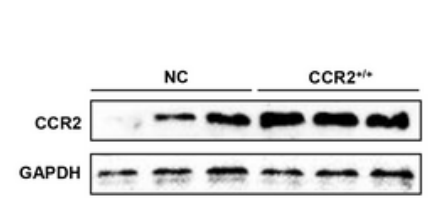

E

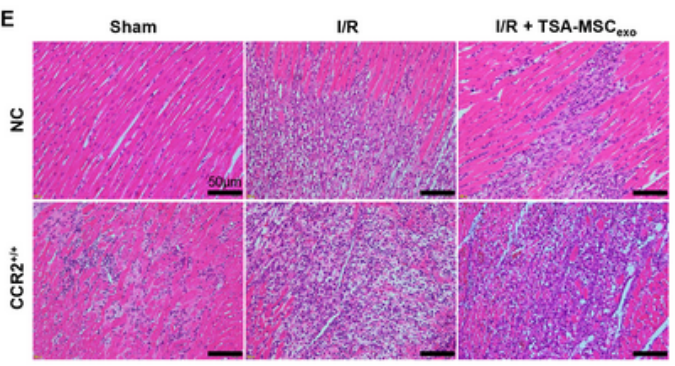

G
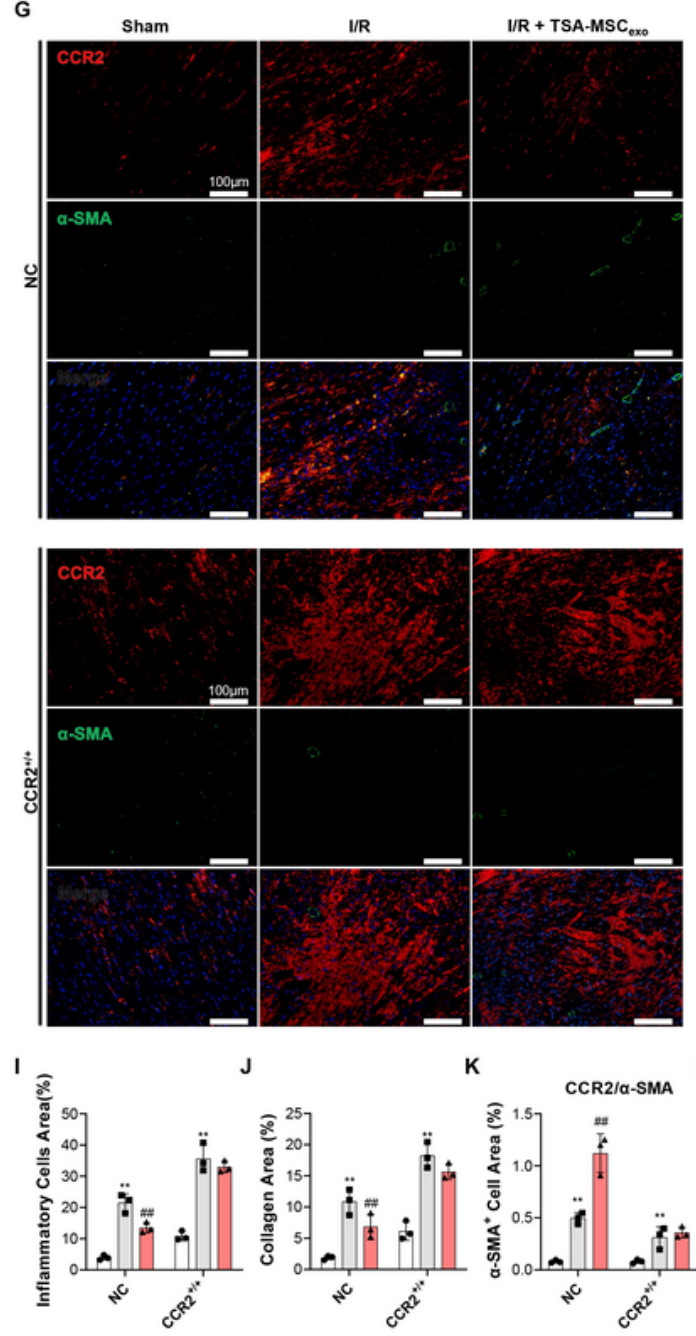

C $\quad$ D

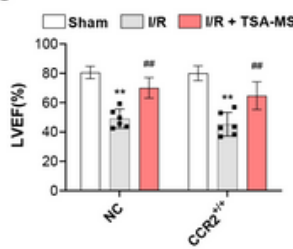

$\square$ Sham $\square$ VR $\square$ UR + TSA-MSC exo
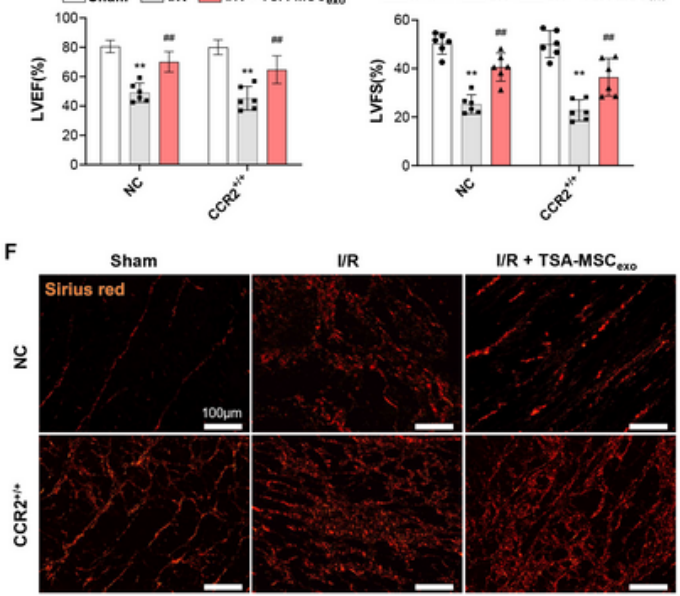

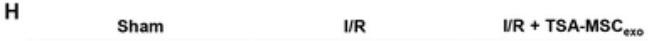
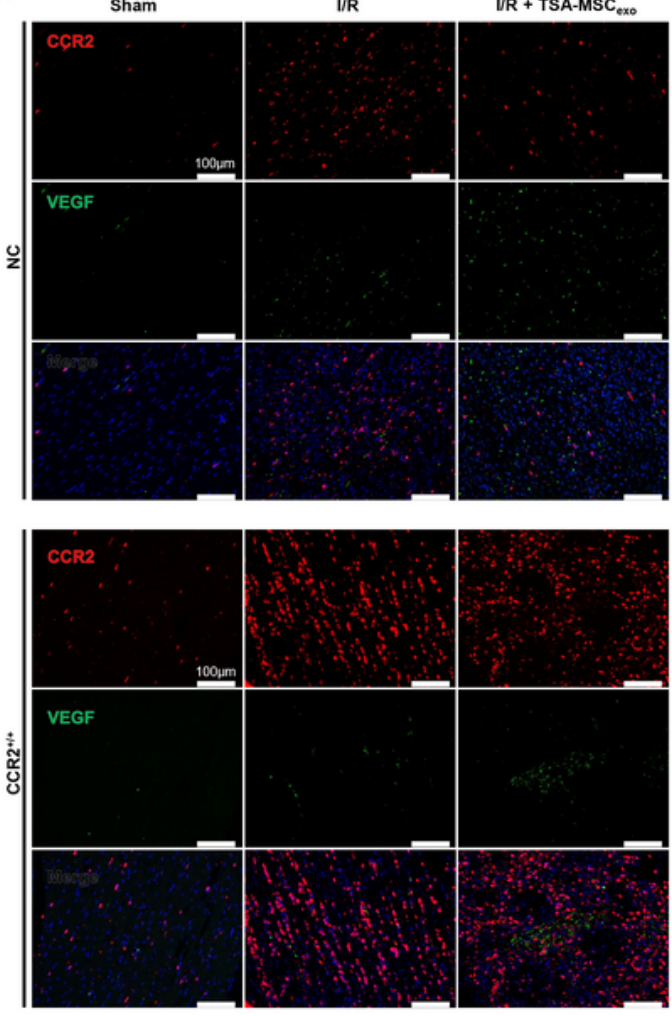

L CCR2/a-SMA ${ }^{\mathrm{M}}$ CCR2/NEGF $\mathrm{N}$ CCR2/NEGF

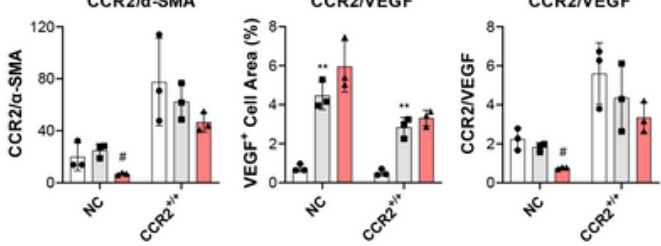

\section{Figure 4}

Overexpression of CCR2 regulates monocyte infiltration and angiogenesis after I/R injury. A

Representative western blot of CCR2 from the heart 8 weeks after transfection of AAV9-CCR2 into the rat myocardium. B Quantitative analysis of CCR2 protein expression in the rat myocardium $(n=3)$. C, D Quantitative analysis of LVEF and LVFS 3 days after reperfusion $(n=6)$. E, F Representative images of HE $($ scale bar $=50 \mu \mathrm{m})$ and Sirius Red (scale bar $=100 \mu \mathrm{m})$ staining of a cross-section of the heart 3 days 
after reperfusion. G Representative images of CCR2 and a-SMA immunofluorescence of heart tissue sections 3 days after reperfusion (scale bar $=100 \mu \mathrm{m}$ ). H Representative images of CCR2 and VEGF immunofluorescence of heart tissue sections 3 days after reperfusion (scale bar $=100 \mu \mathrm{m}$ ). I-N Quantification of inflammatory cell infiltration, collagen deposition, CCR2 and a-SMA immunofluorescence, CCR2 and VEGF immunofluorescence $(n=3)$. Data are expressed as mean \pm SD. **, $p<0.01$ compared with the sham group; $\#, p<0.05, \# \#, p<0.01$ compared with the I/R group.

A

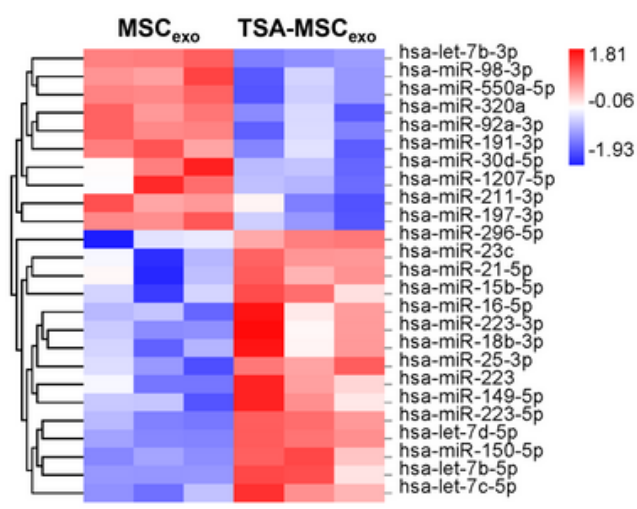

E

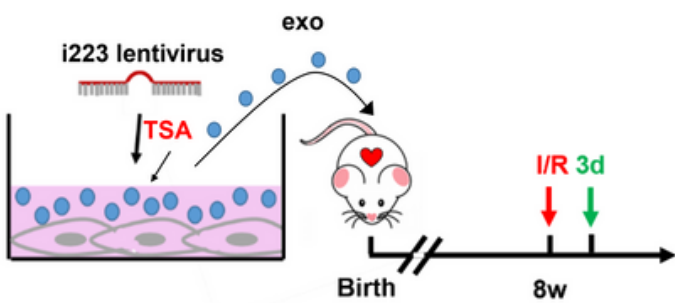

G

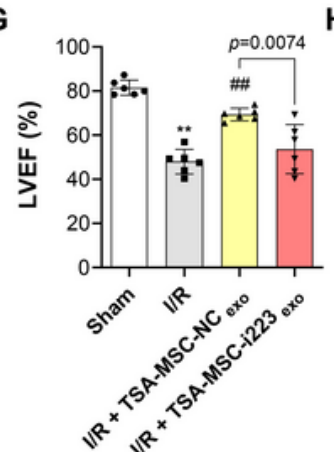

K

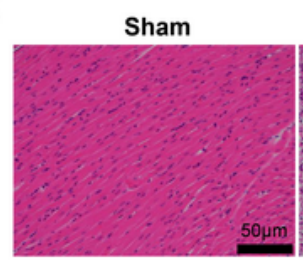

H I

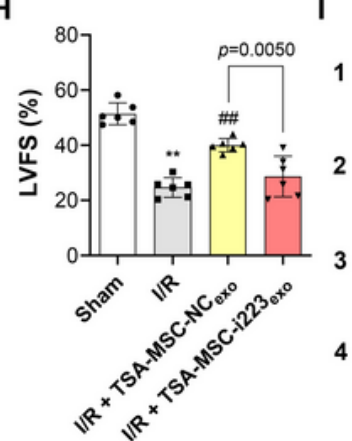

B

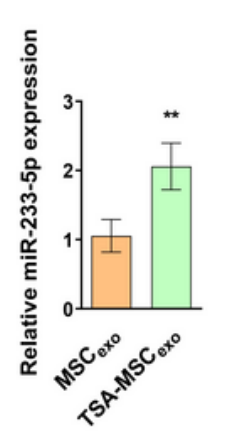

F

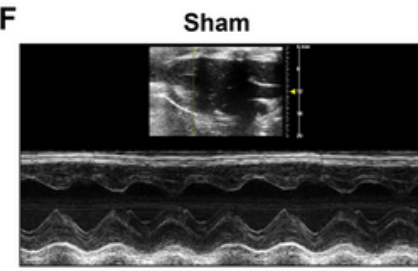

I/R + TSA-MSC-NC

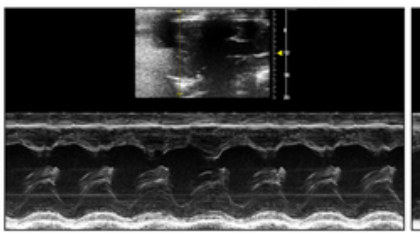

D
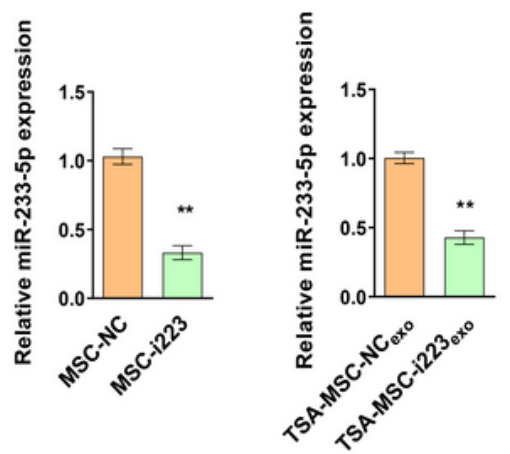

I/R

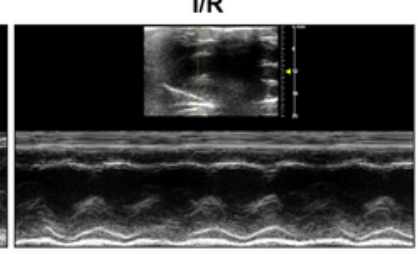

I/R + TSA-MSC-i223

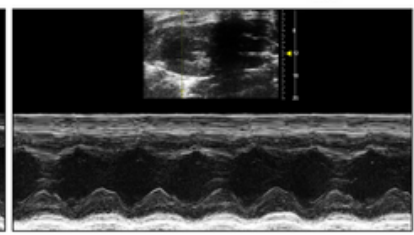

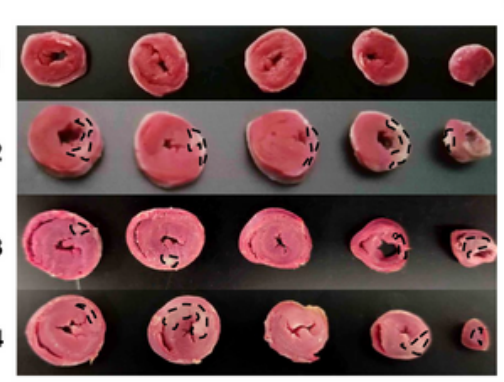
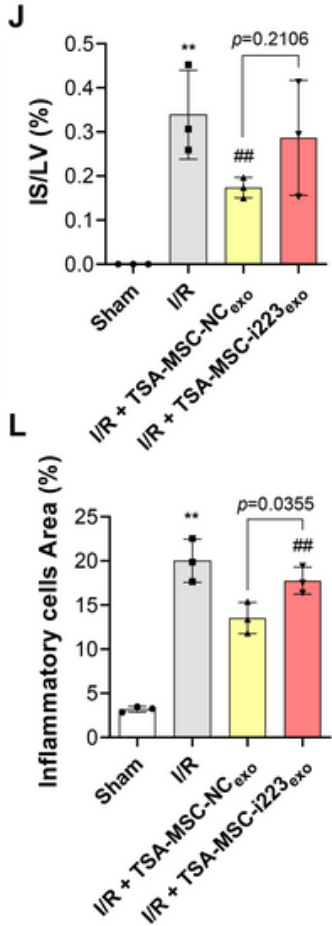
Participation of miR-223-5p in the improvement of TSA-MSCexo-mediated cardiac function and myocardial injury. A Sequencing analysis of miRNA in MSCexo and TSA-MSCexo $(n=3)$. B Relative miR223-5p expression in MSCexo and TSA-MSCexo $(n=3)$. C Relative miR-233-5p expression in MSCs transfected with i223 and NC lentivirus $(n=3)$. D Relative miR-233-5p expression in exosomes isolated after the i223 and NC lentivirus were transfected into MSCs treated with TSA $(n=3)$. Expression of miR223-5p was normalized to U6. E Schematic overview of miR-223-5p inhibitory lentivirus transfected into MSCs, treated with TSA, isolated exosomes, and injected into the rat heart in situ. F Representative images of M-mode two-dimensional ultrasound 3 days after reperfusion. G, H Quantitative analysis of LVEF and LVFS 3 days after reperfusion $(n=6)$. I Representative images of TTC staining of heart sections 3 days after reperfusion. 1: Sham, 2: I/R, 3: TSA-MSC-NCexo, 4: TSA-MSC-i233exo. J Quantification of the percentage of infarct size $(n=3) . K$, L Representative images and quantification of HE staining of a crosssection of the heart 3 days after reperfusion (scale bar $=50 \mu \mathrm{m}$ ). Data are expressed as mean $\pm S D . * \star, p$ $<0.01$ compared with the sham group; \#\#, $p<0.01$ compared with the I/R group. 


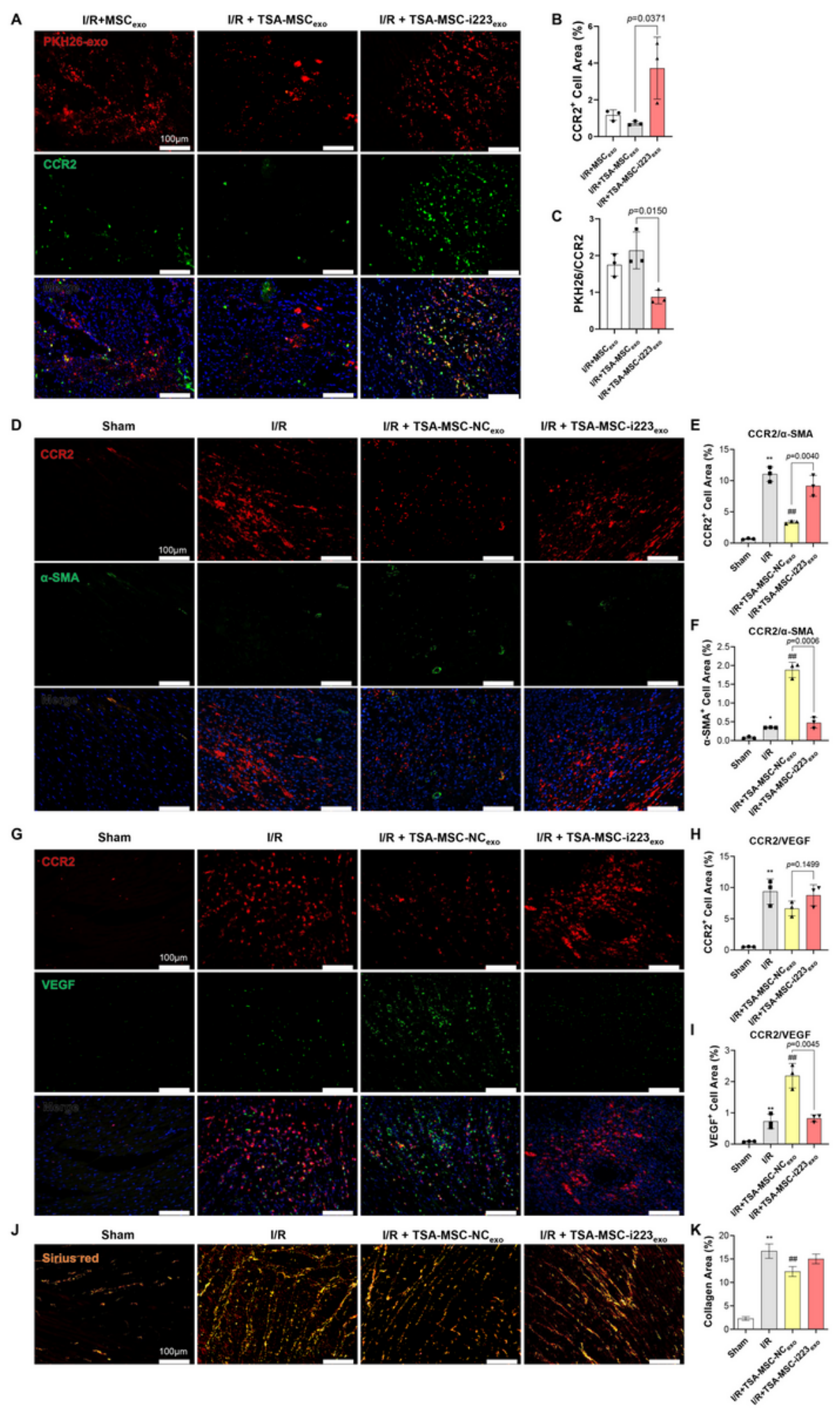

\section{Figure 6}

miR-223-5p shuttling by TSA-MSCexo modulated monocyte infiltration and angiogenesis through targeting CCR2. A-C Representative immunofluorescence images and quantification of CCR2 and MSCexo, TSA-MSC-NCexo, TSA-MSC-i223exo (scale bar $=100 \mu \mathrm{m}$ ). D-F Representative images and quantification of CCR2 and a-SMA immunofluorescence of heart tissue sections 3 days after reperfusion (scale bar $=100 \mu \mathrm{m})$. G-I Representative images and quantification of CCR2 and VEGF 
immunofluorescence of heart tissue sections 3 days after reperfusion (scale bar $=100 \mu \mathrm{m}$ ). J, K Representative images and quantification of Sirius Red staining of a cross-section of the heart 3 days after reperfusion (scale bar $=100 \mu \mathrm{m}$ ). Data are presented from three independent experiments. ${ }^{*}, \mathrm{p}<$ $0.05, * *, p<0.01$ compared with the sham group; \#\#, $p<0.01$ compared with the l/R group.
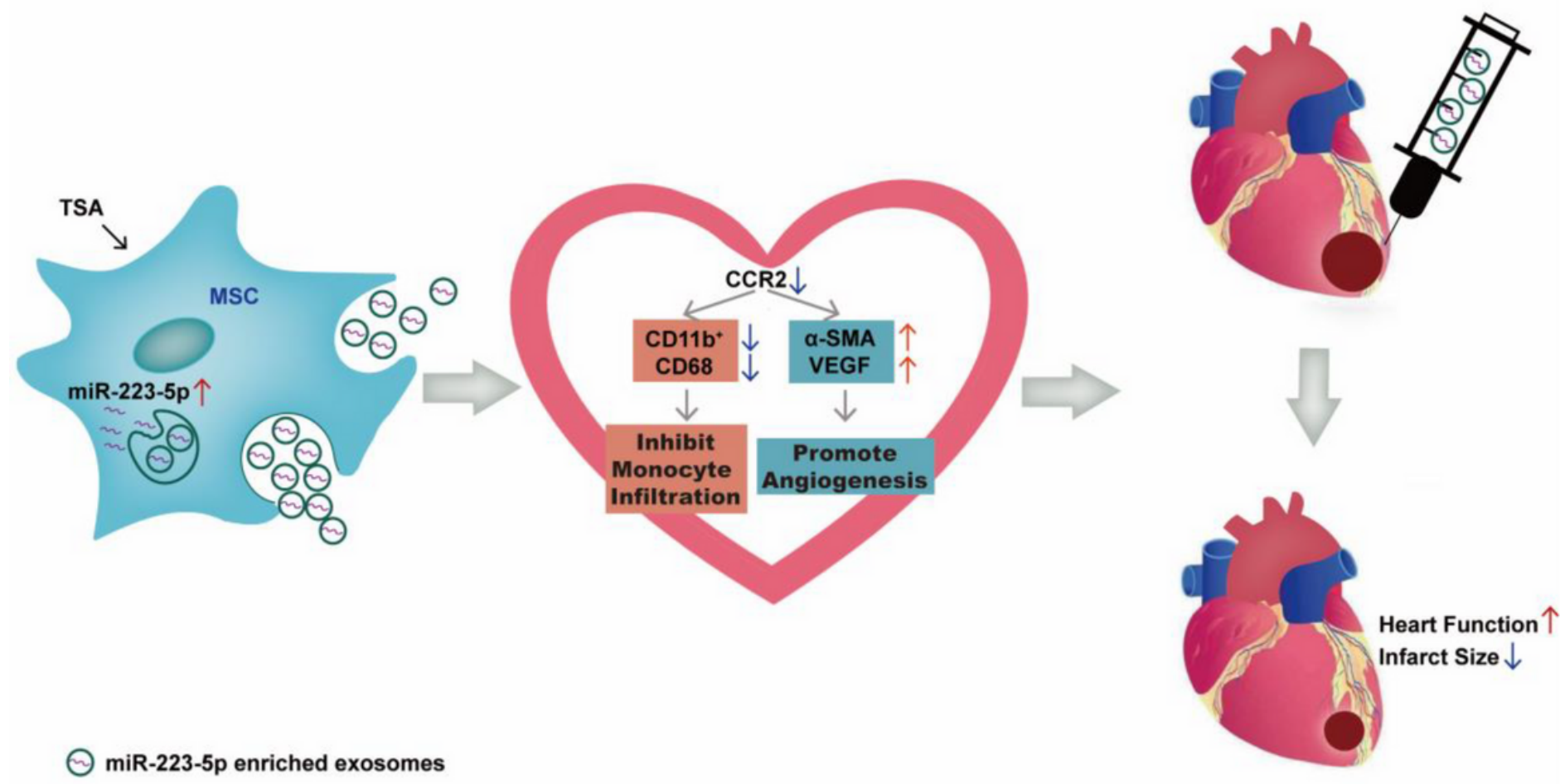

Figure 7

Proposed mechanisms of intramyocardial injection of pretreated with TSA-pretreated MSC-derived exosomes to protect the heart from myocardial I/R injury. TSA increases the production of miR-223-5p in MSCs. miR-223-5p, cargoed in and delivered by TSA-MSCexo to the heart, reduces the infiltration of monocytes and promotes angiogenesis to attenuate inflammation and promote myocardial tissue repair thus ameliorating myocardial I/R injury by inhibiting the activation of CCR2.

\section{Supplementary Files}

This is a list of supplementary files associated with this preprint. Click to download.

- figS1.tif 
- figS2.tif

- figS3.tif

Page 31/31 